BULLETIN (New Series) OF THE

AMERICAN MATHEMATICAL SOCIETY

Volume 36, Number 3, Pages 345-381

S 0273-0979(99)00788-0

Article electronically published on June 23, 1999

\title{
UNIVERSAL FAMILIES AND HYPERCYCLIC OPERATORS
}

\author{
KARL-GOSWIN GROSSE-ERDMANN
}

\section{Contents}

Introduction

Part I. General Theory

1. Universal families

1a. The Universality Criterion

1b. The set of universal elements

1c. Universality in linear spaces

1d. Universal series

2. Hypercyclic operators

2a. The Hypercyclicity Criterion

$2 \mathrm{~b}$. The set of hypercyclic vectors

2c. Derived hypercyclicity

2d. Existence of hypercyclic operators

Part II. Specific Universal Families and Hypercyclic Operators

3. The real analysis setting

3a. Universal power and Taylor series

3b. Universal primitives

3c. Universal orthogonal series

3d. Universal series for convergence a.e.

3e. Further real universalities and hypercyclicities

4. The complex analysis setting

4a. Universal and hypercyclic composition operators

4b. Holomorphic monsters

4c. Hypercyclic differential operators

4d. Universal power and Taylor series

4e. Universal matrices

5. Hypercyclic operators in classical Banach spaces

6. A concrete universal object: the Riemann zeta-function

References

Bibliography

Received by the editors August 4, 1998, and in revised form May 26, 1999.

1991 Mathematics Subject Classification. Primary 47A99, 54H99; Secondary 47-02, 54-02.

Key words and phrases. Universal families, hypercyclic operators.

This survey grew out of a talk given by the author on the occasion of the 60th birthday of Prof. Dr. W. Beekmann at the Fernuniversität Hagen, November 13, 1997.

(C)1999 American Mathematical Society 


\section{INTRODUCTION}

Analysis is the study of limiting processes. Not every limiting process, of course, converges, but examples have been found where processes diverge in a maximal way. Such an extreme behaviour is often linked with the phenomenon of universality, which constitutes the topic of this survey.

The first case of a universality was observed by Fekete [Pál14] in 1914. He showed that there exists a (formal) real power series $\sum_{n=1}^{\infty} a_{n} x^{n}$ on $[-1,1]$ that not only diverges at every point $x \neq 0$ but does so in the worst possible way. Indeed, to every continuous function $g$ on $[-1,1]$ with $g(0)=0$ there exists an increasing sequence $\left(n_{k}\right)$ of positive integers such that $\sum_{n=1}^{n_{k}} a_{n} x^{n} \rightarrow g(x)$ uniformly as $k \rightarrow \infty$. This result becomes even more spectacular when one takes account of Borel's theorem (of 1895) by which every real power series is the Taylor series around 0 of some $C^{\infty}$-function.

Fekete's example of a universal power (or Taylor) series exhibits two aspects of universality that are generally present. Apart from the first aspect of maximal divergence we have as second aspect the existence of a single object which, via a usually countable process, allows us to approximate a maximal class of objects. This suggested the name of universality. It was first employed in 1935 by Marcinkiewicz [Mar35], who proved the existence of a universal 'primitive', a continuous function whose difference quotients can approximate any measurable function in the sense of convergence a.e. (see Section $3 \mathrm{~b}$ ).

In the course of time a great number of universal objects have been discovered. In order to give an example from complex analysis we mention MacLane's result [Mac52] of 1952 on the existence of an entire function $f$ such that to every entire function $g$ there exists an increasing sequence $\left(n_{k}\right)$ of positive integers such that $f^{\left(n_{k}\right)}(z) \rightarrow g(z)$ locally uniformly in $\mathbb{C}$ as $k \rightarrow \infty$. The function $f$ is said to possess universal derivatives.

From an abstract point of view, the phenomenon of universality may be described as follows. We have a topological space $X$ of objects, a topological space $Y$ of elements to be approximated and a family (usually a sequence) $T_{\iota}: X \rightarrow Y(\iota \in I)$ of mappings. Then an object $x$ in $X$ is called universal if every element $y$ in $Y$ can be approximated by certain $T_{\iota} x$, that is, if the set $\left\{T_{\iota} x: \iota \in I\right\}$ is dense in $Y$.

During the last 15 years one particular type of universality, which is already present in MacLane's example, has been studied intensively. In the setting of linear topological spaces, usually a Banach or Hilbert space, one considers sequences $\left(T_{n}\right)$ of operators that are generated by a single continuous linear operator $T$ via iteration, that is, $T_{n}=T^{n}$ for $n \in \mathbb{N}_{0}$. In this special case a universal object is called hypercyclic, a term suggested by the theory of operators in Hilbert spaces and apparently first used in this sense by Beauzamy [Bea86], [Bea87a]. In hypercyclicity, we have besides the two aspects of universality mentioned above a third aspect: Hypercyclicity is a geometric property of the operator $T$ involved. More precisely, an element $x$ is hypercyclic if and only if $T$ has no non-trivial closed invariant subset containing $x$.

One striking feature of universality is that, while one would naively expect it to be a rare phenomenon, quite the opposite is the case. Experience has shown that

- any process in analysis that diverges or behaves irregularly in some cases is likely to produce a universal element. 
Also on the level of objects universality is abundant once it exists. Again, experience has shown that when a process exhibits universality then in most cases

- almost every element is universal

(in the sense of Baire categories). Such a result was first observed by Marcinkiewicz [Mar35] in 1935 for his universal 'primitives' and by Joó [Joó78] in 1978 for the universal orthogonal series of Men'shov and Talalyan (see Section 3c). Since then it has been shown that every 'reasonable' universality enjoys this property.

Thus it appears that universality is a generic phenomenon in analysis.

On this survey. My intention in writing this survey was twofold. As for universality, the number of examples in the literature has increased to such an extent over the last decades that it seemed worthwhile collecting and classifying the results, something that has not been done before. As for hypercyclicity, I believe that with the emergence of the Hypercyclicity Criterion as a basic tool and several recent advances like the results of Ansari-Bernal on the existence of hypercyclic operators, and of Herrero-Bourdon and León-Montes on the existence of large hypercyclic vector manifolds, the basic theory of hypercyclicity has reached a level of maturity where a first survey seems justified.

The survey consists of two parts. Part I presents the theory of universal families (Section 1) and the theory of hypercyclic operators (Section 2). Part II is devoted to collecting and classifying the various universalities and hypercyclicities that have been studied in the literature. Section 3 contains the examples from real analysis, Section 4 those from complex analysis, and Section 5 those from functional analysis. Section 6 deals with the universality of the Riemann zeta-function.

In order to limit the size of this survey I have kept strictly to the two topics referred to in the title. I have excluded, for example, several types of universalities that differ from the one specified in Definition 1 below, such as universal antiderivatives or series that are universal with respect to rearrangements, subseries or signs (but see the end of Section 3d). As for hypercyclicity, I have ignored the related notions of cyclic and supercyclic operators from operator theory (but see Section 2a) and the more general notion of topologically transitive mappings from topological dynamics.

Within this framework I have tried to cover the relevant literature completely. I want to apologise in advance to anyone whose work I have nonetheless overlooked.

\section{ACKNOWLEDGEMENTS}

I would like to thank Luis Bernal, Wolfgang Luh, Alfonso Montes, and Alfredo Peris for their interest in my work and the various ways in which they have contributed to it; and I thank Richard Aron for the opportunity to present a first version of the survey at the Conference on Hypercyclic Operators at Kent State University, March 1998. Comments on the first version and additional references were also supplied by S. I. Ansari, R. Aron, G. Herzog, I. Joó, and A. Laurinčikas, to whom I express my gratitude. In addition, I am grateful to a number of people who have helped to bring this survey more up to date by providing me with their latest preprints.

I would like to take this opportunity to express my special gratitude to Professor Wolfgang Luh for having me introduced to the concept of universality, a concept that spans all parts of analysis and that has led to results that are often beautiful, sometimes surprising, and not rarely both. 


\section{Part I. General Theory}

\section{UNiversal FAMiLies}

1a. The Universality Criterion. As noted in the Introduction the consideration of various examples of universalities in analysis has suggested the following definition [Gro87, 1.2.1]; see also [GeSh87].

Definition 1. Let $X$ and $Y$ be topological spaces and $T_{\iota}: X \rightarrow Y \quad(\iota \in I)$ continuous mappings. Then an element $x \in X$ is called universal (for the family $\left.\left(T_{\iota}\right)_{\iota \in I}\right)$ if the set

$$
\left\{T_{\iota} x: \iota \in I\right\}
$$

is dense in $Y$. The set of universal elements is denoted by $\mathcal{U}=\mathcal{U}\left(T_{\iota}\right)$. The family $\left(T_{\iota}\right)_{\iota \in I}$ is called universal if it has a universal element.

In the special case when the $T_{\iota}$ form a group (under composition) of homeomorphisms on a topological space $X$, the idea of universality is well known in topological dynamics under the name of topological transitivity; see [1, Section 9] and [5, Chapter 5]. In operator theory, when one studies the iterates $\left(T^{n}\right)_{n \in \mathbb{N}_{0}}$ of a (continuous linear) operator $T$, universal elements are usually called hypercyclic; see Section 2. Some authors (see, for example, [GoSh91, 1.1] and $\left[\right.$ Ber99 $\left.{ }^{+}\right]$) have suggested using this term also in the general situation; however, the distinction into universality for general families and hypercyclicity for iterates of single operators seems to best conform with established traditions.

In [Gro87] we have also introduced and studied the more general notion of universality for a non-empty closed subset $A$ of $Y$. An element $x$ in $X$ is called universal (for $\left(T_{\iota}\right)$ ) with respect to $A$ if the closure of the set $\left\{T_{\iota} x: \iota \in I\right\}$ contains $A$. The more general concept is useful in some applications; see, for example, [Gro87, 2.2.11]. In the sequel we restrict ourselves to the standard case of $A=Y$.

We remark at the outset that universality as defined above is a very general concept; in fact it seems to be too general to admit an elaborate theory. For example, any element $x \in X$ becomes universal for $\left(T_{\iota}\right)$ if we shrink $X$ and $Y$ appropriately; also, every $x \in X$ is universal for the constant mappings $T_{\iota} \xi=y_{\iota}$ if the $y_{\iota}$ are chosen to be dense in $Y$. Thus, results on universality in full generality will be rare.

In particular, the most natural problem of characterising when a family of mappings possesses a universal element has not been solved. On the other hand, many results in the literature suggest that whenever a universal element for a particular family exists, the set $\mathcal{U}$ of universal elements may be expected to be huge, in fact residual in the sense of Baire categories. So it is gratifying that residuality of the set $\mathcal{U}$ can be characterised. We refer to [3] for the language of Baire categories.

Theorem 1 (The Universality Criterion). Suppose that $X$ is a Baire space and $Y$ is second-countable. Then the following assertions are equivalent:

(i) The set $\mathcal{U}$ of universal elements is residual in $X$.

(ii) The set $\mathcal{U}$ of universal elements is dense in $X$.

(iii) To every pair of non-empty open subsets $U$ of $X$ and $V$ of $Y$ there exists some $\iota \in I$ with

$$
T_{\iota}(U) \cap V \neq \emptyset .
$$

If one of these conditions holds, then $\mathcal{U}$ is a dense $G_{\delta}$-subset of $X$. 
This criterion is due to the author [Gro87, Satz 1.2.2 and its proof]; see also Joó [Joó89, Lemma 1.2] for '(ii) $\Rightarrow$ (i)' and Godefroy and Shapiro [GoSh91, 1.2]. In the special situation considered in topological dynamics, that is, groups of homeomorphisms or iterates of a single mapping on some topological space $X$, the result is well known; see [1, 9.20], [5, Theorems 5.8, 5.9], and also [Kit82, 2.1], [Bea88, III.5.2]. The proof of the criterion follows easily once it is observed that the set $\mathcal{U}$ can be written as

$$
\mathcal{U}=\bigcap_{n=1}^{\infty} \bigcup_{\iota \in I} T_{\iota}^{-1}\left(V_{n}\right),
$$

where the $V_{n}$ form a base of the topology of $Y$.

Remark 1. In most applications both $X$ and $Y$ are metric spaces. In that case the initial assumptions are satisfied if $X$ is complete and $Y$ is separable. Also, condition (iii) is then equivalent to the following condition that is easier to use in practice:

(iv) To every $x \in X$ and $y \in Y$ there are sequences $\left(x_{k}\right)$ in $X$ and $\left(\iota_{k}\right)$ in I with

$$
x_{k} \rightarrow x \text { and } T_{\iota_{k}} x_{k} \rightarrow y \text {. }
$$

In addition, it clearly suffices to choose the elements $x$ and $y$ from dense subsets of $X$ and $Y$, respectively.

Typically the verification of condition (iii) (or (iv)) reduces to an application of a suitable approximation theorem. For example, in 1929 Birkhoff [Bir29] showed that there exists an entire function $f$ with universal translates; that is, to every entire function $g$ there exists some sequence $\left(a_{n}\right)$ in $\mathbb{C}$ so that

$$
f\left(z+a_{n}\right) \rightarrow g(z)
$$

holds locally uniformly in $\mathbb{C}$ as $n \rightarrow \infty$. In this setting condition (iv) follows easily from Runge's approximation theorem. The Universality Criterion then implies not only the existence of entire functions with universal translates, it even shows that almost every entire function has this property, as was first proved by Duyos-Ruiz [Duy84].

1b. The set of universal elements. So far only very few cases of a universality have come up in the literature where the set of universal elements turned out to be non-residual; see [GoSh91, p. 234], [Gro92, 5.2] and [Hzg95, Section 4]. One explanation of this phenomenon is that under very natural assumptions a kind of topological zero-one law holds: The set $\mathcal{U}$ of universal elements is either empty or residual. The following two results provide settings where this dichotomy holds; a third one will be given in Proposition 6 below. Proposition 1 is due to Peris [Per98], while Proposition 2 is essentially Satz 1.2.6 of [Gro87].

Proposition 1. Let $X$ be a topological space and $T_{\iota}: X \rightarrow X \quad(\iota \in I)$ continuous mappings. Suppose that each $T_{\iota}(\iota \in I)$ has dense range and that the family $\left(T_{\iota}\right)_{\iota \in I}$ is commuting; that is,

$$
T_{\kappa} \circ T_{\iota}=T_{\iota} \circ T_{\kappa} \quad \text { for all } \iota, \kappa \in I .
$$

Then the set $\mathcal{U}$ of universal elements is either empty or dense (resp. residual, if $X$ is a second-countable Baire space). 
Proof. Let $x \in X$ be a universal element. Then the elements $y_{\iota}:=T_{\iota} x(\iota \in I)$ form a dense set in $X$. Fix $\iota \in I$ and let $U \subset X$ be open and non-empty. Since $T_{\iota}$ has dense range and $x$ is universal there is a $\kappa \in I$ with $T_{\kappa} x \in T_{\iota}^{-1}(U)$; hence $T_{\kappa}\left(y_{\iota}\right)=T_{\kappa}\left(T_{\iota} x\right)=T_{\iota}\left(T_{\kappa} x\right) \in U$. This shows that each $y_{\iota}$ is universal. Residuality follows from the Universality Criterion.

Proposition 2. Let $X$ be a Hausdorff topological space without isolated points and $T_{\iota}: X \rightarrow X \quad(\iota \in I)$ continuous mappings. Suppose that, for each $\iota \in I$, every $T_{\kappa}(\kappa \in I)$ with at most finitely many exceptions can be written in the form

$$
T_{\kappa}=T_{\lambda} \circ T_{\iota}
$$

for some $\lambda \in I$. Then the set $\mathcal{U}$ of universal elements is either empty or dense (resp. residual, if $X$ is a second-countable Baire space).

The assumptions in these propositions are satisfied in topological dynamics when the $\left(T_{\iota}\right)$ are a group of homeomorphisms, and in operator theory when one considers a family $\left(T^{n}\right)_{n \in \mathbb{N}_{0}}$ of iterates. In these cases the result is well known; see [1, 9.20], [5, Theorems 5.8, 5.9], [Kit82, 2.1], [GeSh87, 2.1], [Bea88, III.5.1]. In particular we see that since by MacLane's theorem some entire function has universal derivatives, this is so for almost every entire function, as was first observed by Duyos-Ruiz [Duy84].

By our discussion so far it seems that, depending on the ambient space, density or residuality is a mark of a 'true' universality. This suggests the following definitions; cf. Bernal $\left[\right.$ Ber99 $\left.{ }^{+}\right]$.

Definition 2. Let $X$ and $Y$ be topological spaces. Then a family $\left(T_{\iota}\right)_{\iota \in I}$ of continuous mappings $T_{\iota}: X \rightarrow Y$ is called

(i) densely universal if it has a dense set of universal elements,

(ii) generically universal if $X$ is a Baire space and the family has a residual set of universal elements.

An immediate consequence of residuality is the existence of common universal elements, which was first noted by Duyos-Ruiz [Duy84]; see also [GeSh87, p. 282], [Gro87, 1.3.3]. Let $X$ be a fixed Baire space and $T_{\iota}^{(n)}: X \rightarrow Y_{n}\left(\iota \in I_{n}, n \in \mathbb{N}\right)$ continuous mappings.

Proposition 3. If each family $\left(T_{\iota}^{(n)}\right)_{\iota \in I_{n}}(n \in \mathbb{N})$ is generically universal, then there exists an element in $X$ that is universal with respect to each of these families. In fact, the set of common universal elements is residual in $X$.

We have noted above that the universalities of MacLane and Birkhoff are generic universalities. Hence, Proposition 3 immediately asserts the existence of an entire function that is universal in both respects; see [Duy84]. An explicit construction of such a function was given by Blair and Rubel [BlRu84].

In a similar vein one may ask if a known universality can be strengthened by requiring additional properties. It has been studied, for example, if an entire function that is universal in some respect can in addition be zero-free, be univalent or satisfy some growth condition (see Sections $4 \mathrm{a}-4 \mathrm{~d}$ ), or if the coefficients in some universal series can tend to zero with a given speed (see Section 3c). The only general result of this type is due to Herzog [Hzg94, 2.1], who gave a (sufficient) condition under which an improvement of universality is possible. 
In the opposite direction, that is, looking for universalities in larger spaces, a very general result is available; cf. Shapiro [Sha93, p. 111]. For applications we refer to the literature cited in connection with Proposition 9 below.

Proposition 4 (The Universality Comparison Principle). Let $X$ and $Y$ be topological spaces and $T_{\iota}: X \rightarrow Y(\iota \in I)$ continuous mappings. Let $X^{\prime}$ and $Y^{\prime}$ be topological spaces with $X^{\prime} \subset X$ and $Y^{\prime} \subset Y$ such that the mappings $\left.T_{\iota}\right|_{X^{\prime}}: X^{\prime} \rightarrow$ $Y^{\prime}(\iota \in I)$ are well-defined and continuous.

(a) Suppose that $Y^{\prime} \hookrightarrow Y$ continuously and densely. Then we have: If $x \in X^{\prime}$ is universal for $\left(\left.T_{\iota}\right|_{X^{\prime}}\right)$, then it is universal for $\left(T_{\iota}\right)$.

(b) Suppose that $X^{\prime} \hookrightarrow X$ and $Y^{\prime} \hookrightarrow Y$ continuously and densely. Then we have: If $\left(\left.T_{\iota}\right|_{X^{\prime}}\right)$ is a densely universal family, then so is $\left(T_{\iota}\right)$.

Another strengthening of universality has been introduced by Ansari [Ans95] in the case of sequences $\left(T_{n}\right)$.

Definition 3. Let $X$ and $Y$ be topological spaces and $T_{n}: X \rightarrow Y \quad(n \in \mathbb{N})$ continuous mappings. Then the sequence $\left(T_{n}\right)_{n \in \mathbb{N}}$ is called hereditarily universal if every subsequence $\left(T_{n_{k}}\right)_{k \in \mathbb{N}}$ has a universal element.

In addition one may introduce the concepts of densely or generically hereditarily universal sequences as those sequences $\left(T_{n}\right)$ for which each subsequence $\left(T_{n_{k}}\right)$ is densely or generically universal, respectively; cf. Bernal $\left[\right.$ Ber99 $\left.{ }^{+}\right]$. Bès and Peris $\left[\mathrm{BèPe} 99^{+}, 2.6(3)\right]$ have recently obtained a characterisation of mappings $\left(T_{n}\right)$ that have a hereditarily universal subsequence. We refer to our discussion in connection with the corresponding concepts for hypercyclicity; see Definition 5 below.

1c. Universality in linear spaces. Most universalities in the literature live in a linear environment: the spaces $X$ and $Y$ and the corresponding operators are linear. In order to be able to apply the results in Section 1a we shall assume in this section that

$X$ is a Baire topological vector space, $Y$ is a separable metrisable topological vector space and $T_{n}: X \rightarrow Y(n \in \mathbb{N})$ are continuous linear operators.

The field of scalars may be either $\mathbb{R}$ or $\mathbb{C}$. Although the Universality Criterion remains useful in this setting, certain sufficient conditions have turned out to be very effective and easily applicable in practice.

In 1978, Joó [Joó78, Lemma 1] gave a sufficient condition that is useful in the context of universal series; indeed it was the first general condition for universality in the literature. See [Söv77], [Bog78] for related discussions. Kitai [Kit82] in 1982 and Gethner and Shapiro [GeSh87] in 1987 independently found a condition for the universality of iterates of a single operator, the so-called Hypercyclicity Criterion; see Section 2a. The following is a synthesis of these conditions.

Theorem 2 (A Sufficient Condition). Suppose that there are dense subsets $X_{0}$ of $X$ and $Y_{0}$ of $Y$ and (possibly non-linear and discontinuous) mappings $S_{n}: Y_{0} \rightarrow X$ such that

(i) for every $x \in X_{0}, T_{n_{k}} x \rightarrow 0$ for some $\left(n_{k}\right)$,

(ii) for every $y \in Y_{0},\left(S_{n} y\right)$ converges,

(iii) for every $y \in Y_{0},\left(T_{n} \circ S_{n}\right) y \rightarrow y$.

Then the sequence $\left(T_{n}\right)_{n \in \mathbb{N}}$ has a dense $G_{\delta^{-}}$, hence residual, subset of universal vectors. 
In Joó's criterion the mappings $S_{n}$ all coincide, while in the Hypercyclicity Criterion and its variants for sequences of mappings we have $S_{n} y \rightarrow 0$ for every $y \in Y_{0}$; see [GeSh87, 2.3(a)], [God89], [Gro90] and [GoSh91, 1.4]. A related condition was also given by Bernal [Ber94, Theorem A], [Ber96, 2.1].

For the proof of Theorem 2 we use the Universality Criterion. Let $U \subset X$ and $V \subset Y$ be non-empty open subsets. Choose $y \in V \cap Y_{0}$ and let $a=\lim _{n \rightarrow \infty} S_{n} y$. Further choose $x \in U \cap\left(X_{0}+a\right)$, and let $\left(n_{k}\right)$ be a sequence with $T_{n_{k}}(x-a) \rightarrow 0$. Then we have $\xi_{k}:=S_{n_{k}} y-a+x \in U$ and $T_{n_{k}} \xi_{k}=\left(T_{n_{k}} \circ S_{n_{k}}\right) y+T_{n_{k}}(x-a) \in V$ if $k$ is sufficiently large.

Remark 2. Several variants of Theorem 2 are possible. It clearly suffices if the conditions hold for a suitable subsequence $\left(T_{m_{n}}\right)_{n}$. Also, if all the limits in (ii) are zero, then one may weaken (i) to mere convergence (the case $T_{n}=S_{n}=$ id shows that we cannot have mere convergence in both (i) and (ii)). Furthermore, the quantifier ' $\exists\left(n_{k}\right)^{\prime}$ ' can be shifted from (i) to (ii) or (iii).

Another sufficient condition for universality that is based on the existence of a large supply of joint eigenvectors of the $T_{n}$ was recently formulated by Bernal [Ber99b, Theorem 7], [Ber99+]; cf. [GoSh91, Section 5].

In 1945 Men'shov [Men45], [Men47] showed that every trigonometric series is the sum of two universal trigonometric series; cf. Section 3c. Since then a corresponding result has been obtained for various other universalities. In fact, decomposability is an immediate consequence of residuality, as was first shown by the author [Gro87, 1.4.3], and also by Godefroy [BoSh90, p. 46] and Kahane [Nes96, 3.2].

Proposition 5. If $\left(T_{n}\right)_{n}$ is a generically universal family on $X$, then every vector in $X$ is the sum of two universal vectors, that is,

$$
X=\mathcal{U}+\mathcal{U} .
$$

This shows that in general two universal vectors do not add up to a universal vector. Thus it has come as a surprise that, nonetheless, many linear universal families possess 'large' subspaces consisting entirely, apart from 0 , of universal vectors. General results in this direction are due to Montes [Mon96b, 2.2 and following Remarks] and Bernal [Ber99 ${ }^{+}$. We shall discuss this matter in greater detail in connection with hypercyclicity; see Section $2 \mathrm{~b}$.

In the linear setting we have another remarkable and rather curious zero-one law (cf. Propositions 1 and 2) which in addition is widely applicable: If the $T_{n}$ are well-behaved on a dense set and they are badly-behaved on just one element, then they are badly-behaved on most elements; see [Gro87, 1.4.2] and, more generally, [Gro92, 4.1, 4.2].

Proposition 6. If the sequence $\left(T_{n}\right)$ converges pointwise on a dense subset of $X$, then the set $\mathcal{U}$ of universal elements is either empty of residual.

For example, from Fekete's existence of a universal Taylor series and Weierstrass' approximation theorem it follows immediately that almost every $C^{\infty}$-function $f$ on $[-1,1]$ with $f(0)=0$ has a universal Taylor series; cf. [Gro87, 2.1.5] and also [Maz37].

To our knowledge the results we have discussed or mentioned so far cover everything that is known about universality in full generality. 
1d. Universal series. An inspection of the universalities collected in Part II of this survey shows that a large majority of them falls into one of two big classes: universal series and hypercyclic vectors. A theory of universal series has not been developed yet, but one may state a general result on the existence of universal series when there are no restrictions on the coefficients. This result is in the background of every construction of an unrestricted universal series, for example in [Pál14], [Sie38], [Tal57] or [GoWa60], and it is formulated for particular spaces in Seleznev, Motova and Volokhin [SMV77, Theorem 1] and Ivanov [Iva86], [Iva89, Theorem 5]; a similar characterisation when there is in addition a certain restriction on the coefficients is due to Khavinson [Kha61a, Theorems 2 and 4].

Proposition 7. Let $X$ be a metrisable topological vector space and $\left(x_{n}\right)$ a sequence in $X$. Then the following assertions are equivalent:

(i) There exists a universal series $\sum_{n=1}^{\infty} a_{n} x_{n}$ in $X$; that is, there are scalars $a_{n}(n \in \mathbb{N})$ such that to every element $x$ in $X$ there is an increasing sequence $\left(n_{k}\right)$ of positive integers with

$$
\sum_{n=1}^{n_{k}} a_{n} x_{n} \rightarrow x \quad \text { in } X .
$$

(ii) For every $n_{0} \in \mathbb{N}, \operatorname{span}\left\{x_{n}: n \geq n_{0}\right\}$ is dense in $X$.

We refer to Section 3d for a stronger result in a more restricted setting.

On the other hand, hypercyclicity, which constitutes the other big class of universalities, has recently attracted much attention and has by now developed into an extended theory that links universality with operator theory. To this theory we turn next.

\section{HYPERCYCLIC OPERATORS}

2a. The Hypercyclicity Criterion. Hypercyclicity is the study of the universality of sequences $\left(T_{n}\right)$ that are generated by a single mapping $T: X \rightarrow X$ via iteration, that is, $T_{n}=T^{n}$ for $n \in \mathbb{N}_{0}$. In addition, attention is restricted to the linear setting; the field $\mathbb{K}$ of scalars may be either $\mathbb{R}$ or $\mathbb{C}$.

Definition 4. Let $X$ be a topological vector space and $T: X \rightarrow X$ a continuous linear operator. Then a vector $x \in X$ is called hypercyclic (for $T$ ) if its orbit

$$
\left\{T^{n} x: n \in \mathbb{N}_{0}\right\}
$$

is dense in $X$. The set of vectors that are hypercyclic for $T$ is denoted by $\mathcal{H C}=$ $\mathcal{H C}(T)$. The operator $T$ is called hypercyclic if it has a hypercyclic vector.

Correspondingly, the operator $T$ will be called densely hypercyclic $\left[\mathrm{Ber} 9^{+}\right]$if its set of hypercyclic vectors is dense, generically hypercyclic if this set is residual with $X$ being a Baire space. It is a consequence of Proposition 1 that every hypercyclic operator is even densely hypercyclic.

Strictly speaking, the first operator to be proved hypercyclic in the literature was the operator of differentiation in the space $H(\mathbb{C})$ of entire functions (MacLane [Mac52], 1952). But Birkhoff's much earlier proof of the universality of translates ([Bir29], 1929) had essentially already shown the hypercyclicity of each translation operator $T_{a} f(z)=f(z+a), a \neq 0$, in $H(\mathbb{C})$; cf. Luh [Luh78]. The first examples of hypercyclic operators in a Banach or Hilbert space setting appeared in 1969 and are due to Rolewicz [Rol69], who also initiated the general theory of hypercyclicity. 
It took almost another 15 years before the theory was taken up by Kitai [Kit82]; see also [HKR85], Beauzamy [Bea86], [Bea87a], [Bea87b], [Bea88] and Gethner and Shapiro [GeSh87] in the mid-80's. During the last ten years it has been studied intensively.

Recently, the notion of hypercyclicity was extended to include the universality of strongly continuous semigroups $(T(t))_{t \geq 0}$ of bounded linear operators on a Banach space; see [DSW97], [DVW97], [Ema97], [Ema98].

The term 'hypercyclic' was apparently introduced in its present meaning by Beauzamy [Bea86], [Bea87a]. It was motivated by the well-known notion of cyclicity in operator theory. A vector $x \in X$ is called cyclic if the linear span of its orbit $\left\{T^{n} x: n \in \mathbb{N}_{0}\right\}$ is dense in $X$. Accordingly, $x$ is called supercyclic if the set $\left\{\lambda T^{n} x: \lambda \in \mathbb{K}, n \in \mathbb{N}_{0}\right\}$ is dense in $X$. The notion of hypercyclicity corresponds to the invariant subset problem as that of cyclicity does to the invariant subspace problem: The operator $T$ has no non-trivial closed invariant subset if and only if every vector $x \neq 0$ is hypercyclic for $T$.

We remark that not only hypercyclicity but also cyclicity and supercyclicity can be regarded as particular instances of universalities. Indeed, a vector $x$ is cyclic for $T$ if and only if it is universal for the family $\left(\sum_{k=0}^{n} a_{k} T^{k}\right)_{n \in \mathbf{N}_{0} ; a_{0}, \ldots, a_{n} \in \mathbb{K}}$; it is supercyclic for $T$ if and only if it is universal for the family $\left(\lambda T^{n}\right)_{\lambda \in \mathbb{K}, n \in \mathbb{N}_{0}}$.

The following results are specialisations of results in Section 1. We recall that an F-space is a completely metrisable topological vector space. We shall assume that the underlying space is separable, which is clearly necessary for any hypercyclic operator to exist.

Theorem 3. Let $X$ be a separable $F$-space and $T$ a continuous linear operator on $X$. Then the following assertions are equivalent:

(i) $T$ is hypercyclic.

(ii) $T$ is generically hypercyclic.

(iii) To each pair of non-empty open subsets $U$ and $V$ of $X$ there exists some $n \in \mathbb{N}_{0}$ with

$$
T^{n}(U) \cap V \neq \emptyset .
$$

If one of these conditions holds, then the set $\mathcal{H C}(T)$ of hypercyclic vectors is a dense $G_{\delta}$-subset of $X$.

This characterisation is well known in topological dynamics [5, Theorem 5.9]; see also [Kit82, 2.1], [Bea88, III.5.2] and [DSW97, 2.2]. In concrete situations it is often easier to verify the following equivalent condition:

(iii) To every $x, y \in X$ there are sequences $\left(x_{k}\right)$ in $X$ and $\left(n_{k}\right)$ in $\mathbb{N}_{0}$ such that

$$
x_{k} \rightarrow x \text { and } T^{n_{k}} x_{k} \rightarrow y,
$$

where it in fact suffices to take the $x$ and $y$ from (possibly different) dense subsets.

While this result characterises hypercyclicity, the following (a priori) sufficient condition has proved extremely useful in applications. It is due, independently, to Kitai [Kit82, 1.4] and Gethner and Shapiro [GeSh87, 2.2, 2.3(b)]; cf. [GoSh91, 1.5]. We state here the criterion in the weak form due to Bès and Peris [BèPe99 $\left.{ }^{+}\right]$; it is a special case of Theorem 2 .

Theorem 4 (The Hypercyclicity Criterion). Let $X$ be a separable F-space and $T$ a continuous linear operator on $X$. Suppose that there are dense subsets $X_{0}$ and $Y_{0}$ 
of $X$, an increasing sequence $\left(n_{k}\right)$ of positive integers and (possibly non-linear and discontinuous) mappings $S_{n_{k}}: Y_{0} \rightarrow X$ such that

(i) for every $x \in X_{0}, T^{n_{k}} x \rightarrow 0$,

(ii) for every $y \in Y_{0},\left(S_{n_{k}} y\right) \rightarrow 0$,

(iii) for every $y \in Y_{0},\left(T^{n_{k}} \circ S_{n_{k}}\right) y \rightarrow y$.

Then the operator $T$ is hypercyclic.

Under the conditions stated in the theorem the operator $T$ is said to satisfy the Hypercyclicity Criterion $\left(\right.$ for $\left.\left(n_{k}\right)\right)$. If, in particular, one may choose $n_{k}=k$, then $T$ is said to satisfy the Hypercyclicity Criterion for the full sequence. In order to avoid confusion one should note that the name 'Hypercyclicity Criterion' has been attached to various forms of the criterion in the literature. For example, in many cases it is required that the mappings $S_{n_{k}}$ arise from a single mapping $S: Y_{0} \rightarrow Y_{0}$ via iteration, that is, $S_{n_{k}}:=S^{n_{k}}$, and that, instead of (iii), we have $T \circ S=\operatorname{id}_{Y_{0}}$, the identity on $Y_{0}$.

Remark 3. It follows from Theorem 2 and Remark 2 that the conditions in the criterion can be weakened in various directions.

A related, weaker condition was obtained by Bernal [Ber94, Theorem A], [Ber96, 2.1]; see also [DSW97, 2.3]. Godefroy and Shapiro [God89], [GoSh91, Sections 4, 5] have observed that a generous supply of eigenvectors often ensures hypercyclicity. A corresponding criterion was formulated by Bernal [Ber99b, Theorem 7]; see also Flytzanis [Fly95, §3]. In the context of strongly continuous semigroups of operators Desch, Schappacher and Webb [Web95, 4.2], [DSW97, §3] have obtained sufficient conditions for hypercyclicity in terms of the spectrum of the infinitesimal generator.

To see the Hypercyclicity Criterion at work we consider MacLane's universality again. In that case, $T$ is the operator of differentiation on $H(\mathbb{C})$ with $T^{n}$ converging pointwise to zero on the dense subset $\mathcal{P}$ of polynomials. If the operator $S$ is defined by $S f(z)=\int_{0}^{z} f(\zeta) d \zeta$, then $S_{n}:=S^{n}$ converges pointwise to zero on the same set $\mathcal{P}$. It then follows that $T$ is hypercyclic.

Salas and Herrero [Sal91, Remark 2(b)], [Her91] have shown that there are hypercyclic operators (on Hilbert space) that do not satisfy the Hypercyclicity Criterion for the full sequence, but so far no operator has been found that does not satisfy the Hypercyclicity Criterion in its general form. This has led to the following question.

Problem 1 (León, Montes [LeMo99+], Bès, Peris [BèPe99+]). Does every hypercyclic operator (on Hilbert space, on a Banach space) satisfy the Hypercyclicity Criterion?

Bès and Peris $\left[\right.$ BèPe99 $\left.9^{+}, 2.14\right]$ have recently shown that on Fréchet spaces every chaotic operator, that is, every hypercyclic operator with a dense set of periodic points, satisfies the Hypercyclicity Criterion. They have also established the equivalence of Problem 1 with another basic problem on hypercyclicity; see Problem 3 below.

It is a consequence of Theorem 2 that if the operator $T$ satisfies the Hypercyclicity Criterion for the full sequence, then any subsequence $\left(T^{n_{k}}\right)_{k}$ has a universal vector [GeSh87, 2.3(a)]. Also, if $T$ and $U$ are two operators on separable F-spaces $X$ and $Y$, respectively, that both satisfy the Hypercyclicity Criterion for the full sequence, then the direct sum operator $T \oplus U$ on $X \oplus Y$ is hypercyclic [Sal91, Remark 2(b)]. Both assertions become false for general hypercyclic operators; see Section 2c. 
$2 \mathrm{~b}$. The set of hypercyclic vectors. We first note the following immediate consequences of residuality; cf. Propositions 3 and 5.

Proposition 8. Let $X$ be a separable F-space.

(a) If $T$ is a hypercyclic operator on $X$, then every vector in $X$ is the sum of two hypercyclic vectors, that is,

$$
X=\mathcal{H C}+\mathcal{H C} .
$$

(b) If $T_{n}(n \in \mathbb{N})$ are hypercyclic operators on $X$, then there exists a vector in $X$ that is hypercyclic for each $T_{n}$. In fact, the set of common hypercyclic vectors is a dense $G_{\delta^{-}}$, hence residual, subset of $X$.

Assertion (a) indicates that hypercyclicity is not a linear phenomenon (even if one disregards the zero vector which is never hypercyclic anyway). So the question arose if, nonetheless, there exist hypercyclic operators that possess a higher-dimensional hypercyclic vector manifold, that is, a linear subspace which, apart from zero, consists entirely of hypercyclic vectors. This was answered positively by Beauzamy [Bea86], [Bea90], see also [Bea87a], who exhibited an operator on complex Hilbert space with a dense invariant hypercyclic vector manifold. The key idea was to take a hypercyclic vector $x$ and study the (obviously dense and invariant) subspace

$$
\operatorname{span}\left\{x, T x, T^{2} x, \ldots\right\}=\{p(T) x: p \text { a complex polynomial }\} \text {. }
$$

Godefroy and Shapiro [God89, GoSh91] showed that for a large number of operators this subspace produces a hypercyclic vector manifold. Herrero [Her91] and Bourdon [Bou93] then independently proved that this is indeed so for every hypercyclic operator on complex Hilbert space. As noted in [HeLe93, Bemerkung 4], Bourdon's proof also works in arbitrary complex locally convex spaces. Finally, Bès [Bès99] obtained the corresponding result for real spaces, thus answering a question posed by Ansari [Ans97].

Theorem 5 (Herrero, Bourdon, Bès). Every hypercyclic operator on a (real or complex) locally convex space has a dense invariant hypercyclic vector manifold.

In a different direction one may ask if a given hypercyclic operator has a closed infinite-dimensional hypercyclic vector manifold. This problem has recently been discussed by Montes and co-workers in a series of papers [BeMo95b], [Mon96b], [Mon97], [LeMo97], [Mon98a], [LeMo99 $\left.{ }^{+}\right]$, [GoLM99 ${ }^{+}$. In particular, [GoLM99'] characterises the operators on separable complex Banach spaces that have the stated property if the operator satisfies the Hypercyclicity Criterion (or, equivalently, if it is hereditarily hypercyclic with respect to some $\left(n_{k}\right)$; cf. Definition 5 and Theorem 9 below). See also [Mon96b, 2.2 and following Remarks], [LeMo99 ${ }^{+}$, Theorem 2.1].

Theorem 6 (González, León, Montes). Let $T$ be a continuous linear operator on a separable complex Banach space $X$. If $T$ satisfies the Hypercyclicity Criterion, then the following assertions are equivalent:

(i) The operator $T$ has a closed infinite-dimensional hypercyclic vector manifold.

(ii) There is a closed infinite-dimensional subspace $X_{0}$ of $X$ and an increasing sequence $\left(n_{k}\right)$ of positive integers such that for every $x \in X_{0}$

$$
T^{n_{k}} x \rightarrow 0 .
$$

(iii) The essential spectrum of $T$ intersects the closed unit disk. 
The authors ask if the condition that $T$ satisfies the Hypercyclicity Criterion can be replaced by the mere assumption that $T$ is hypercyclic [LeMo97], [LeMo99+], $\left[\right.$ GoLM99 ${ }^{+}$.

Chan [Cha99] has given an elegant new proof of part of the León-Montes result for a Hilbert space $H$ by studying the hypercyclicity, in the strong operator topology, of the induced left multiplication operator $L_{T}$ on the operator algebra $B(H)$ defined by $L_{T}(V)=T V$.

A closed hypercyclic vector manifold cannot be invariant - for in that case it has to be dense - unless the whole space is a hypercyclic vector manifold. This leads us back to the basic problem that had motivated much of the early work on hypercyclic operators: Do there exist operators on Hilbert space for which every non-zero vector is hypercyclic? This is the same as asking for an operator with no non-trivial closed invariant subset, in particular with no such subspace. In a remarkable paper, Read [Rea88] has given a positive answer in a Banach space setting, notably for the space $l^{1}$.

Theorem 7 (Read). There exists a continuous linear operator on $l^{1}$ for which every non-zero vector is hypercyclic.

The initial problem has remained open; cf. [Kit82, Introduction], [Bea88, p. 75].

Problem 2 (Edelstein, Radjavi). Does there exist a continuous linear operator on (separable infinite-dimensional) Hilbert space for which every non-zero vector is hypercyclic?

It is remarkable that the answer is positive in some pre-Hilbert spaces [Bea86], [Bea90], cf. [GoSh91, p. 230], or if the operator is allowed to be discontinuous [HNRR86, Theorem 6], or if one considers continuous but non-linear mappings $T$ with $T(0)=0$. This last result, a positive answer to a question posed in [HNRR86, Question 5], is due to Peris [Per98]; he observes that the existence of such a mapping $T$ follows directly from Read's theorem and the Anderson-Kadec theorem by which any two infinite-dimensional separable Banach spaces are homeomorphic (cf. [4, $\S 6])$.

2c. Derived hypercyclicity. In this section we turn to the following general problem: If an operator $T$ is derived from a hypercyclic operator in a specific way, does it follow that $T$ itself is hypercyclic?

One way of obtaining new operators is by making the underlying space smaller or larger; we refer to our discussion in connection with Proposition 4 above in the context of general universal families. For hypercyclic operators we have the following result by Shapiro [Sha93, p. 111].

Proposition 9 (The Hypercyclicity Comparison Principle). Let $T$ be a continuous linear operator on a topological vector space $X$. Let $Y$ be a topological vector space such that $Y \hookrightarrow X$ continuously and densely. If $\left.T\right|_{Y}: Y \rightarrow Y$ is a well-defined, continuous and hypercyclic operator, then $T$ is hypercyclic (on $X)$; in particular, $T$ has a hypercyclic vector that belongs to $Y$.

The principle shows why it can be useful to search for hypercyclicities on smaller spaces. For applications we refer to [Sha93, pp. 123-124], [ChSh91, pp. 1432-1433] and $\left[\right.$ Bon99 $\left.{ }^{+}\right]$. A generalisation of the principle was recently given by Martínez and Peris $\left[\mathrm{MaPe} 99^{+}\right]$. 
Since condition (iii) in Theorem 3 reads the same for $T$ and $T^{-1}$, if the latter exists, we have the following; cf. [Kit82, 2.2], [Bea88, III.5.3], and see also [HeKi92] and [DSW97, 2.5].

Proposition 10. If $T$ is an invertible continuous linear operator on an F-space, then $T$ is hypercyclic if and only if $T^{-1}$ is.

If $T$ is hypercyclic, does it follow that each iterate $T^{n}(n \in \mathbb{N})$ is hypercyclic? This question, posed by Kitai [Kit82, 2.13], was recently answered by Ansari [Ans95, Theorem 1], [Ans97, Note 3]; see also [Bou96].

Theorem 8 (Ansari). Let $T$ be a hypercyclic operator on a locally convex space. Then $T^{n}$ is hypercyclic for every $n \in \mathbb{N}$, and $T$ and $T^{n}$ have the same set of hypercyclic vectors.

For a stronger result in the setting of Fréchet spaces we refer to Bès and Peris [BèPe99 ${ }^{+}$, Section 4], who also show that if $T$ satisfies the Hypercyclicity Criterion, then so does each power $T^{n}(n \in \mathbb{N})\left[\right.$ BèPe99 $\left.9^{+}, 2.7\right]$.

It follows from the theorem that for every hypercyclic operator $T$ each subsequence of the special form $\left(T^{k n}\right)_{k \in \mathbb{N}_{0}}$ with $n \in \mathbb{N}$ has a universal element. This has suggested the following definition [Ans95].

Definition 5. Let $\left(n_{k}\right)$ be an increasing sequence of positive integers. A continuous linear operator $T$ on a topological vector space is called hereditarily hypercyclic with respect to $\left(n_{k}\right)$ if every subsequence $\left(T^{n_{k_{j}}}\right)_{j}$ of $\left(T^{n_{k}}\right)_{k}$ has a universal element. It is called hereditarily hypercyclic if it is hereditarily hypercyclic with respect to the full sequence $\left(n_{k}\right)=(k)$.

Hereditarily hypercyclic operators have also been called strongly hypercyclic [Sha93, p. 125], [BoSh97, p. 37]. Some authors $\left[\mathrm{BèPe} 99^{+}\right],\left[\mathrm{GoLM} 99^{+}\right]$require of a hereditarily hypercyclic operator only that it be hereditarily hypercyclic with respect to some sequence $\left(n_{k}\right)$, a property that might be shared by all hypercyclic operators; cf. Problem 3 and Theorem 9 below.

We remark that, as a consequence of Theorem 2, any operator on a separable F-space that satisfies the Hypercyclicity Criterion for a sequence $\left(n_{k}\right)$ is even hereditarily hypercyclic with respect to this sequence; cf. [GoSh91, Remark (a) to 1.5], [BèPe99+, 2.6(1)].

In addition, an operator $T$ will be called densely (generically) hereditarily hypercyclic if each subsequence $\left(T^{n_{k}}\right)_{k}$ of $\left(T^{n}\right)_{n}$ is densely (generically) universal. It is a consequence of Proposition 1 that every hereditarily hypercyclic operator is densely hereditarily hypercyclic, and it is even generically hereditarily hypercyclic if the underlying space is a separable F-space; cf. [Ber99 ${ }^{+}$, $\left[\mathrm{GoLM}^{+} 9^{+}, 2.2\right]$. On Banach spaces, Herzog and Lemmert [HeLe98] have obtained a nice geometric property of hereditarily hypercyclic operators.

Herzog and Lemmert also note that on the space $\omega$ of all real sequences every hypercyclic operator is hereditarily hypercyclic. This is not so, however, for arbitrary hypercyclic operators on Hilbert space $H$. Indeed, Salas and Herrero [Sal91, Remark 2(a)], [Her91] have shown that there exist hypercyclic operators $T_{1}$ and $T_{2}$ on $H$ such that the direct sum $T_{1} \oplus T_{2}$ on $H \oplus H$ is not hypercyclic. On the other hand, it follows easily from the Universality Criterion that the direct sum of a (hereditarily) hypercyclic operator and a hereditarily hypercyclic operator on any $F$-space, say, is again (hereditarily) hypercyclic; cf. [HeLe98, Proposition 1]. Thus neither $T_{1}$ nor $T_{2}$ is hereditarily hypercyclic. 
The Salas-Herrero example provided the first case of two hypercyclic operators whose direct sum is not hypercyclic, thus answering an earlier question of Herrero. It has remained open, however, if the two operators can coincide.

Problem 3 (Herrero [Her92, Problem 1]). Let $T$ be a hypercyclic operator on Hilbert space $H$. Does it follow that the operator $T \oplus T$ on $H \oplus H$ is hypercyclic?

The answer is clearly positive if $T$ satisfies the Hypercyclicity Criterion. By a remarkable result of Bès and Peris $\left[\mathrm{BèPe} 99^{+}, 2.3\right]$ this is in fact also a necessary condition.

Theorem 9 (Bès, Peris). Let $T$ be a continuous linear operator on a separable $F$ space $X$. Then the following assertions are equivalent:

(i) The operator $T \oplus T$ on $X \oplus X$ is hypercyclic.

(ii) $T$ satisfies the Hypercyclicity Criterion.

(iii) $T$ is hereditarily hypercyclic with respect to some sequence $\left(n_{k}\right)$.

This also shows that Problems 1 and 3 are equivalent and that they are equivalent to the question if every hypercyclic operator is hereditarily hypercyclic with respect to some sequence $\left(n_{k}\right)$.

Herrero has posed another interesting problem. One might generalise the notion of hypercyclicity by demanding only the existence of finitely many vectors whose orbits, taken together, form a dense set; cf. [Her92], [Sal95].

Definition 6. A continuous linear operator $T$ on a topological vector space $X$ is called multi-hypercyclic if there are vectors $x_{1}, \ldots, x_{m}$ in $X$ such that the set $\left\{T^{n} x_{j}: n \in \mathbb{N}_{0}, 1 \leq j \leq m\right\}$ is dense in $X$.

For example, if $T$ is hypercyclic, then one may consider the vectors $x_{j}=T^{j-1} x$, $j=1, \ldots, n$, for any hypercyclic vector $x$ to see that each operator $T^{n}$ is multihypercyclic. Now, if $X$ is a Hilbert space, Ansari (cf. Theorem 8) has shown not only that $T^{n}$ itself is hypercyclic, but that indeed one of the vectors $x_{j}$ is hypercyclic for $T^{n}$. Herrero conjectured that this is so for any multi-hypercyclic operator on Hilbert space.

Problem 4 (Herrero [Her92, Conjecture 1]). Let $T$ be a multi-hypercyclic operator on Hilbert space. Does it follow that $T$ is hypercyclic? Is one of the $x_{j}$ a hypercyclic vector for $T$ ?

Positive answers to the first question have been obtained for several classes of operators by Salas [Sal95] and Bourdon and Shapiro [BoSh97, p. 36]. Multihypercyclic operators are also studied in Miller [Mil97].

2d. Existence of hypercyclic operators. It is an amusing fact that hypercyclicity is a purely infinite-dimensional phenomenon.

Proposition 11. No linear operator on a finite-dimensional space is hypercyclic.

This result is due to Rolewicz [Rol69, p. 17]; see also [Kit82, 1.2], [ChSh91, pp. 1445-1446] and [Bès99, Lemma 2]. We indicate here a linear algebra proof of this basic fact following Kitai [Kit82, 1.2]. First we may suppose that we are dealing with the Euclidean $\mathbb{R}^{N}$ or $\mathbb{C}^{N}$; in addition we may assume that the matrix of the operator $T$ under investigation is in Jordan normal form with respect to the standard basis. Then we either have $T^{n}\left(x_{1}, \ldots, x_{N}\right)=\left(\ldots, \lambda^{n} x_{N}\right)$ for some 
(real or complex) scalar $\lambda$ or $T^{n}\left(x_{1}, \ldots, x_{N-1}, x_{N}\right)=\left(\ldots, \lambda^{n}\left(\cos (n \varphi) x_{N-1}-\right.\right.$ $\left.\left.\sin (n \varphi) x_{N}\right), \lambda^{n}\left(\sin (n \varphi) x_{N-1}+\cos (n \varphi) x_{N}\right)\right)$ for some real numbers $\lambda$ and $\varphi$, and all $n \in \mathbb{N}_{0}$. This shows that no vector $\left(x_{1}, \ldots, x_{N}\right)$ can be hypercyclic.

A second restriction on a topological vector space to carry a hypercyclic operator is that it clearly has to be separable. In 1969, Rolewicz [Rol69] asked if in the setting of Banach spaces these are the only restrictions, that is, if every separable infinitedimensional Banach space carries a hypercyclic operator. This was recently and independently answered in the affirmative by Ansari [Ans97] and Bernal [Ber99a]. In fact, Ansari's proof allows her to extend this result to much larger classes of topological vector spaces. In particular she shows that every Fréchet space with an equicontinuous biorthogonal system admits a hypercyclic operator [Ans97, Theorem 1(c)]. Bonet and Peris [BoPe98] were able to remove the latter restriction.

Theorem 10 (Ansari, Bernal, Bonet, Peris). Every separable infinite-dimensional Fréchet space carries a hypercyclic operator.

Each of the proofs relies in an essential way on an earlier result of Salas [Sal95] on the hypercyclicity of some specific operators on $l^{1}$.

Bonet and Peris obtain the same result also for Hausdorff countable inductive limits of separable Banach spaces, provided that one of the steps is dense in the whole space. On the other hand, we shall see that Theorem 10 does not remain true on arbitrary (complete) locally convex spaces.

Remark 4. (a) In the class of complete LB-spaces not every separable infinitedimensional space carries a hypercyclic operator. To see this we consider the space $\varphi$ of terminating scalar sequences, that is, $\varphi=\bigcup_{N=1}^{\infty} E_{N}$ with $E_{N}=\left\{\left(x_{k}\right): x_{k}=\right.$ 0 for $k>N\}$; the space is endowed with its natural (locally convex) inductive topology. Suppose now that $T: \varphi \rightarrow \varphi$ is an operator with a hypercyclic vector $x$. We claim that one of the subspaces $E_{N}$ contains infinitely many $T^{n} x$. Otherwise, since all $T^{n} x$ are non-zero, we can find numbers $\varepsilon_{k}>0(k \in \mathbb{N})$ such that no $T^{n} x$ belongs to the set $U=\left\{\xi \in \varphi:\left|\xi_{k}\right|<\varepsilon_{k}, k \in \mathbb{N}\right\}$. This is a contradiction since $U$ is a neighbourhood of 0 in $\varphi$; cf. $[2,4.1,6.6]$. Hence there are some $N \in \mathbb{N}$ and an increasing sequence $\left(n_{k}\right)$ with $T^{n_{k}} x \in E_{N}$ for all $k$. We can then find some $m \in \mathbb{N}$ such that $T^{n_{m}} x \in \operatorname{span}\left\{T^{n_{1}} x, \ldots, T^{n_{m-1}} x\right\} \subset \operatorname{span}\left\{x, T x, \ldots, T^{n_{m}-1} x\right\}=: F$. This implies that $\left.T\right|_{F}: F \rightarrow F$ is a hypercyclic operator on a finite-dimensional space, contradicting Proposition 11. Hence no operator on $\varphi$ is hypercyclic. This also answers in the negative the first question in [Ans97, Problem 3]. The same result was also obtained by Bonet and Peris [BoPe98], who even show that no operator on $\varphi$ can be supercyclic.

(b) The second question in [Ans97, Problem 3] asks if the spaces $L^{p}=L^{p}[0,1]$ $(0<p<1)$ admit hypercyclic operators. This is indeed so; one may consider composition operators $C_{\varphi}: L^{p} \rightarrow L^{p}, C_{\varphi} f=f \circ \varphi$ for suitable $\varphi$. For example, if $\varphi(t)=t / 2$ for $t \in\left[0, \frac{1}{2}\right]$ and $\varphi(t)=3 / 2 t-1 / 2$ for $t \in\left[\frac{1}{2}, 1\right]$, then $C_{\varphi}$ is a well-defined invertible continuous linear operator on any $L^{p}$ that satisfies the Hypercyclicity Criterion when $X_{0}=Y_{0}$ is taken as the subspace of continuous functions $f$ with $f(0)=f(1)=0$.

\section{Problem 5.}

(a) Characterise the topological vector spaces that support a hypercyclic operator. 
(b) Does every separable infinite-dimensional $F$-space (quasi-Banach space) support a hypercyclic operator?

Studying polynomials instead of linear operators, Bernardes $\left[\right.$ Brn99+ ${ }^{+}$has recently shown that for $m \geq 2$ no continuous $m$-homogeneous polynomial on any Banach space can have a dense orbit. By Peris $\left[\right.$ Per99 $\left.{ }^{+}\right]$, however, there exist even chaotic continuous $m$-homogeneous polynomials for any $m \in \mathbb{N}$ on the Fréchet space $H(\mathbb{C})$ of entire functions.

After the Ansari-Bernal result the following problem seems to have a better chance of having a positive answer.

Problem 6 (Halperin, Kitai, Rosenthal [HKR85]). Let $A$ be a countable linearly independent subset of a separable Banach space $X$. Does there exist an operator $T$ on $X$ such that the orbit $\left\{T^{n} x: n \in \mathbb{N}_{0}\right\}$ of some $x \in X$ under $T$ contains $A$ ?

The three authors have shown that the answer is positive in Hilbert space.

Additional open problems on general hypercyclicity can be found in [Rol69], [Mil97], [Ber99a] and [Cha99 ${ }^{+}$.

With this we end our survey of the theory of hypercyclicity. Many authors have studied this theory from an operator theoretic point of view. Topics under investigation include

- classes of operators that contain no hypercyclic operator (for example, compact or normal operators); see [Kit82], [ChSh91, Section 4], [HeLe93], [Mat93, 2.8-2.11], [DSW97, §3], [BoPe98];

- density of the set of hypercyclic operators in the operator algebra $\left[\right.$ Cha99 $\left.{ }^{+}\right]$;

- periodic orbits of hypercyclic operators and chaos; see [HeWa90], [GoSh91, Section 6], [Sal91], [Her92], [TiLi95], [TiLu96], [DSW97], [BèPe99+], [Bon99+], $\left[\mathrm{MaPe} 99^{+}\right]$;

- hypercyclicity and the spectrum of operators; see [Kit82], [Bea87b], [HeWa90], [ChSh91, Section 4], [Her91], [Her92], [Mat93], [Fly95], [Mat95], [DSW97], $\left[\mathrm{LeMo}^{+}\right]$, [GoLM99 $\left.{ }^{+}\right]$;

- hypercyclicity and functional calculus; see [HeSc94], [Scm97], [MiMi99], [CaWa98].

For the study of concrete hypercyclic operators in a Banach or Hilbert space setting we refer to Section 5 .

To date, hypercyclicity has made its appearance in two textbooks: in [Bea88] and [Sha93].

\section{Part II. Specific Universal Families and Hypercyclic Operators}

In the second part of the survey we describe and classify the universalities and hypercyclicities that have been studied in the literature. In each section we state one typical result; we stress that it is not necessarily the most general result available.

\section{The Real Analysis SETting}

3a. Universal power and Taylor series. We have already encountered Fekete's universal power series of 1914 in the Introduction. Its partial sums approximate any continuous function uniformly on $[-1,1]$ if it vanishes at 0 ; see Pál [Pál14], [Pál15]. Further existence proofs (on [0,1]) are due to Mazurkiewicz [Maz37] and Sierpiński [Sie38]; cf. [GeOl64, pp. 74-75]. Seleznev [Sel51], Lorentz [Lor53, p. 46] and Luh [Luh78], [Luh79b] obtained power series that are universal for locally 
uniform convergence in $\mathbb{R} \backslash\{0\}$. We remark that by Borel's theorem each of these power series is a Taylor series around 0 of some $C^{\infty}$-function. A modification of Fekete's proof leads to a universal power (Taylor) series on all of $\mathbb{R}$; see [Gro87, 2.1.4, 2.1.5].

Theorem 11. There exists a function $f \in C^{\infty}(\mathbb{R})$ with $f(0)=0$ whose Taylor series $\sum_{n=1}^{\infty} a_{n} x^{n}$ around 0 is universal in the sense that to every function $g \in C(\mathbb{R})$ with $g(0)=0$ there is an increasing sequence $\left(n_{k}\right)$ of positive integers with

$$
\sum_{n=1}^{n_{k}} a_{n} x^{n} \rightarrow g(x) \quad \text { locally uniformly in } \mathbb{R} .
$$

The set of such functions $f$ is residual in the space of all functions in $C^{\infty}(\mathbb{R})$ that vanish at 0.

In [Gro87] it was also shown that if we only want local uniform approximability on $(-1,1)$, the coefficients in a universal Taylor series may be chosen to be integers.

Luh [Luh70, Luh74a] has obtained universal power series of radius of convergence 1 with approximation in $\mathbb{R} \backslash[-1,1]$ under a (very general) summability matrix.

We also refer to Section $4 \mathrm{~d}$ for related results in the complex plane.

3b. Universal primitives. In 1935 Marcinkiewicz [Mar35] not only proved the existence of what he called a universal primitive, he was also the first to use the word 'universal' in such a context and the first to show that a set of universal elements is residual.

Theorem 12 (Marcinkiewicz). Let $\left(h_{n}\right)$ be a sequence of real numbers with $h_{n} \rightarrow$ 0 . Then there exists a continuous function $f \in C[0,1]$ such that to every measurable function $g$ on $[0,1]$ there is an increasing sequence $\left(n_{k}\right)$ of positive integers with

$$
\frac{f\left(x+h_{n_{k}}\right)-f(x)}{h_{n_{k}}} \rightarrow g(x) \text { a.e. in }[0,1] .
$$

The set of such functions $f$ is residual in $C[0,1]$.

Several authors have obtained strengthenings, generalisations and variants of this result. Tuy [Tuy59], [Tuy60] shows that the values $g(x)$ can be derived numbers in a stronger sense, a martingale version is due to Lamb [Lam74], Aversa and Carrese [AvCa83] obtain an ( $n$-dimensional) version for interval functions, Grande [Gra84] gives a Banach space-valued generalisation, Cater [Cat89] replaces the difference quotient $(f(x+h)-f(x)) / h$ by certain higher-order difference quotients, and Gan and Stromberg [GaSt94] obtain the generalisation of Marcinkiewicz' theorem for functions $f:[0,1]^{n} \rightarrow \mathbb{R}^{m}$. Krotov [Kro91] shows that a universal primitive can in addition enjoy some smoothness properties; in particular it can have continuum many points of differentiability in any subinterval.

Joó [Joó89] studied the problem when one replaces a.e.-convergence by convergence in $L^{p}$. He showed the existence of a universal primitive $f$ in the space $L^{1}$ (on any bounded open set in $\mathbb{R}^{N}$ ) with approximation of functions $g$ in $L^{p}$ for any $p<1$. Several authors showed that one cannot choose $p \geq 1$ here; see [BoSö87], [Buc87], [Hor87]. Some related problems are raised in [Joó89], [Joó91].

See [Bru78] and [Str81] for the inclusion of Marcinkiewicz' result in textbooks.

3c. Universal orthogonal series. One of the most remarkable universalities is due to Men'shov, who in 1945 proved the existence of a universal trigonometric series with coefficients tending to zero; see [Men45], [Men47]. In 1957, Talalyan 
[Tal57] showed that this remains true if one replaces the trigonometric system by any complete orthonormal system in $L^{2}$.

Theorem 13 (Men'shov, Talalyan). Let $\left(\varphi_{n}\right)$ be a complete orthonormal system in $L^{2}[0,1]$. Then there exists a series $\sum_{n=1}^{\infty} a_{n} \varphi_{n}$ with $a_{n} \in \mathbb{R}$ such that to every measurable function $f$ on $[0,1]$ there exists an increasing sequence $\left(n_{k}\right)$ of positive integers such that

$$
\sum_{n=1}^{n_{k}} a_{n} \varphi_{n}(t) \rightarrow f(t) \quad \text { a.e. in }[0,1] .
$$

In addition, one may have $a_{n} \rightarrow 0$.

We remark that convergence a.e. can be replaced here, equivalently, by convergence in measure. The depth of the theorem lies in the fact that the coefficients $a_{n}$ may tend to zero. Without this requirement the result is considerably simpler and holds in great generality, as will be seen in the next section.

In 1950, Kozlov [Koz50] essentially showed that there exists a universal trigonometric series such that, in addition, if $f$ is continuous on $[0,1]$, then the convergence in $(*)$ can be locally uniform in $(0,1)$; see also [Gro87, proof of 2.1.10] and [Nes96, proof of 3.1]. However, as noted in [Bar61, Chapter XV, §6], the coefficients of such a series cannot tend to zero; see also [LuLu85]. For a generalisation of Kozlov's result we refer to Nestoridis and Melas $\left[\mathrm{Nes}_{9} 9^{+}\right]$, [MeNe99 ${ }^{+}, 5.3$ with proof].

Men'shov had also shown that every trigonometric series with coefficients tending to zero is the sum of two universal trigonometric series of this type. A stronger result (cf. Proposition 5) is due to Joó [Joó78], who proved for every complete orthonormal system that in the space $c_{0}$ of zero sequences the set of sequences $\left(a_{n}\right)$ that generate a universal series is residual.

Extensions and variants of the Men'shov-Talalyan theorem have been obtained by several authors: orthogonal series that can approximate any measurable function $f$ that lies between two preassigned measurable functions $F$ and $G$ with $F \leq G$, but no others (Men'shov [Men48], [Men50]; see also [Men54], [Men63], [Men64], Talalyan [Tal59a], Körner [Kör89]), types of convergence other than convergence a.e. (Ivanov [Iva81], [Iva83a], [Iva83b], [Iva89], Buczolich [Buc87], Joó [Joó89]), summability instead of convergence (Men'shov [Men63], [Men64], Buczolich [Buc87]), and the multi-variable case (Dzagnidze [Dza64], Ivanov [Iva81], [Iva83a]). Some interesting investigations, motivated by Problem 10 in [Tal60c], are devoted to the problem of how fast the coefficients $a_{n}$ of a universal orthogonal series can converge to zero; see Pogosyan [Pog76], Ivanov [Iva81], [Iva83a], [Iva83b], [Iva89] and Körner [Kör89].

Krotov and Bakhshetsyan [Kro74], [Kro75], [Kro77], [BaKr81] have studied some of these problems for the (non-orthogonal) Faber-Schauder system. In [Kro91] Krotov also showed that a universal trigonometric series can be a Fourier-Stieltjes series.

For treatments of universal orthogonal series in textbooks we refer to [KaSt58], [Ale60] and [Bar61].

3 d. Universal series for convergence a.e. Let $\left(\varphi_{n}\right)$ be an arbitrary sequence of measurable functions on $[0,1]$. The existence of a universal series

$$
\sum_{n=1}^{\infty} a_{n} \varphi_{n}
$$


in the sense of convergence a.e. (or, equivalently, in measure) can be asserted in great generality when no restriction is imposed on the scalar sequence $\left(a_{n}\right)$. For the existence of such a series it is clearly necessary that the linear span of the $\varphi_{n}$ be dense in the space $M[0,1]$ of measurable functions on $[0,1]$ endowed with the (metrisable) topology of convergence in measure. Talalyan [Tal57] showed that this condition is already sufficient. Goffman and Waterman [GoWa60] (see also [GoPe65, $5.5]$ ) deduced this result from the fact that the topological dual of $M[0,1]$ is trivial. Similar results for other spaces of measurable functions are due to Goffman and Waterman [GoWa72] and Ivanov [Iva86], [Iva89, Corollary 1]. In effect we have the following characterisation, which should be compared with Proposition 7.

Theorem 14 (Goffman, Waterman, Ivanov). Let $X$ be a (real or complex) metrisable topological vector space and $\left(x_{n}\right)$ a sequence in $X$. Suppose that $X$ has trivial dual. Then the following assertions are equivalent:

(i) There exists a universal series $\sum_{n=1}^{\infty} a_{n} x_{n}$ in $X$; that is, there are scalars $a_{n}(n \in \mathbb{N})$ such that to every element $x$ in $X$ there is an increasing sequence $\left(n_{k}\right)$ of positive integers with

$$
\sum_{n=1}^{n_{k}} a_{n} x_{n} \rightarrow x \quad \text { in } X .
$$

(ii) The linear span of $\left\{x_{n}: n \in \mathbb{N}\right\}$ is dense in $X$.

A particular universal series with unrestricted coefficients was obtained by Edge [Edg70]. For additional results on general universal series and sequences see Hejduk [Hej89] and Chkhaidze [Chk77].

To end our discussion of universal series, we remark that a large number of investigations deals with universalities for series that differ from the one considered so far: we refer to series that are universal with respect to rearrangements, subseries or signs. These, however, are not universalities in the sense of Section 1 and are therefore excluded from this survey. The papers [Tal59b], [Tal60a], [Tal60b], [Tal60c], $[\mathrm{Pog} 75$, Remark 1] contain results that compare these kinds of universalities with the one studied in this and the previous section.

3e. Further real universalities and hypercyclicities. As we shall see in the following sections there is a large number of universalities and hypercyclicities for holomorphic functions. It seems natural to look for corresponding results for harmonic functions on $\mathbb{R}^{N}$. So far there are few results in that direction. Dzagnidze [Dza69] and, independently, Armitage and Gauthier [ArGa96, Section 5] have extended Birkhoff's theorem on universal translates (cf. Section 4a) to harmonic functions, while Aldred and Armitage [AlAr98a], [AlAr98b], [Arm99 ${ }^{+}$] have extended MacLane's theorem on universal derivatives (cf. Section 4c) and also obtained the least-possible rate of growth for the corresponding universal functions. We seem to have here a large potential for future investigations.

In the space $C^{\infty}\left(\mathbb{R}^{N}\right)$, the universality of translates was shown by Duyos-Ruiz ([Duy83, Corollary 1], $N=1$ ); the hypercyclicity of any (partial) differential operator with constant coefficients that is not a scalar multiple of the identity was obtained by Todorov ([Tod85], $N=1$; see also [ToKo80]) and Godefroy and Shapiro $\left[\right.$ GoSh91, 5.5(b)]. Bonet $\left[\right.$ Bon99 $\left.{ }^{+}\right]$studies the hypercyclicity of convolution and partial differential operators on spaces of ultradifferentiable or real analytic functions and on spaces of ultradistributions. 
Several authors have recently studied the phenomenon of hypercyclicity for continuous semigroups of linear operators that are generated by the solutions of partial differential equations like the transport equation or the heat equation; see [PrAz92], [Web95], [DSW97], [DVW97], [Hzg97], [Ema97] and [Ema98]. We have here another very promising new direction in hypercyclicity.

Herzog [Hzg91] (see also [Hzg88]) has exhibited a universality phenomenon in Lagrange interpolation. On $[0,1]$ there exists a matrix of nodes and a continuous function $f$ so that the Lagrange interpolation polynomials $P_{n}$ of $f$ with respect to these nodes are dense in each space $L^{p}[0,1], 1 \leq p<\infty$.

\section{The Complex Analysis Setting}

4a. Universal and hypercyclic composition operators. In 1929, Birkhoff [Bir29] proved the existence of an entire function $f$ with universal translates, that is, with the property that to every entire function $g$ there exists a sequence $\left(a_{n}\right)$ in $\mathbb{C}$ with $a_{n} \rightarrow \infty$ such that

$$
f\left(z+a_{n}\right) \rightarrow g(z) \quad \text { locally uniformly in } \mathbb{C} .
$$

In terms of the translations $\tau_{a}(z)=z+a$, which are particular conformal automorphisms of $\mathbb{C}$, Birkhoff's theorem asserts the universality of the family $\left(C_{\tau_{a}}\right)_{a \in \mathbb{C}}$ of composition operators $C_{\tau_{a}}: H(\mathbb{C}) \rightarrow H(\mathbb{C}), C_{\tau_{a}}(f)=f \circ \tau_{a}$. In 1941, Seidel and Walsh [SeWa41] obtained an analogue for non-Euclidean translates in the unit disk $\mathbb{D}$.

The residuality of the corresponding sets of universal functions was shown by Duyos-Ruiz [Duy84], Gethner and Shapiro [GeSh87] and the author [Gro87, 2.3].

Variants and strengthenings of the theorems of Birkhoff and Seidel and Walsh were found by Heins [Hei55], Luh [Luh78], [Luh79a] and Shapiro [Sha93, pp. 111, 123], while Gauthier [Gau94, pp. 248-249] gave a new proof of Birkhoff's theorem. Duyos-Ruiz [Duy83] obtained functions with universal translates of arbitrarily slow transcendental growth (see also [ChSh91, Corollary to 2.1]). Luh, Martirosian and Müller [LMM98], [LMM99 ${ }^{+}$showed that the Taylor series of a universal function can have certain gaps. Functions with additional universality properties are constructed in [BIRu84], [Duy84], [Luh96] and [LMM98].

Recently, Luh [Luh93] and Bernal and Montes [BeMo95a], [BeMo95b], [Mon96a] have considered composition operators $C_{\varphi}: H(O) \rightarrow H(O), C_{\varphi}(f)=f \circ \varphi$ on general open subsets $O$ of $\mathbb{C}$. Bernal and Montes have identified those sequences $\left(\varphi_{n}\right)$ of conformal automorphisms of $O$ that lead to universal functions. In order to state their result we recall that a sequence $\left(\varphi_{n}\right)$ is said to act properly discontinuously on $O$ if for every compact subset $K$ of $O$ there is some $n \in \mathbb{N}$ with $K \cap \varphi_{n}(K)=\emptyset$. The authors call such a sequence $\left(\varphi_{n}\right)$ simply a run-away sequence. In addition, let $\mathbb{C}^{*}=\mathbb{C} \backslash\{0\}$.

Theorem 15 (Bernal, Montes). Let $O \subset \mathbb{C}$ be an open set that is not conformally equivalent to $\mathbb{C}^{*}$, and let $\left(\varphi_{n}\right)$ be a sequence of automorphisms of $O$. Then there exists a function $f \in H(O)$ for which the set $\left\{f \circ \varphi_{n}: n \in \mathbb{N}\right\}$ is dense in $H(O)$ if and only if $\left(\varphi_{n}\right)$ is a run-away sequence. In case of existence the set of such functions $f$ is residual in $H(O)$.

The run-away sequences of automorphisms of $\mathbb{C}$ and $\mathbb{D}$ have been determined by Bernal and Montes [BeMo95a], and Montes [Mon96a] has characterised those open 
sets for which run-away sequences exist and hence for which universal functions in the sense of Theorem 15 can be found.

The excluded case of the punctured plane $\mathbb{C}^{*}$ was first studied by Zappa [Zap88] and taken up by Bernal and Montes [BeMo95a], [BeMo95b]. It turns out that Theorem 15 cannot be extended to $\mathbb{C}^{*}$, but that every run-away sequence leads to some weaker type of universality; cf. [BeMo95a, 3.3]. Versions of Zappa's result on $G L_{n}(\mathbb{C})$ and $S L_{n}(\mathbb{C})$ are due to Abe and Zappa [AbZa99 ${ }^{+}$], [Abe99 ${ }^{+}$.

Extensions in other directions are due to Chee [Che79], Godefroy and Shapiro [God89], [GoSh91, 5.1], Abe and Zappa [Abe97], [AbZa99+'] and León [Leó99] (the $N$-dimensional case); to Aron and Bès [ArBè99] (entire functions on a Banach space); to Zappa [Zap88] and Montes [Mon98b] (Riemann surfaces); and to Herzog [Hzg95], Bernal and Calderón [BeCa99 ${ }^{+}$] (operators of the form $T(f)=\Phi(D) f \circ \varphi$, where $D$ is the differentiation operator). For a related kind of universality see [Mil93].

Several authors have studied the hypercyclicity of composition operators in Hilbert or Banach spaces of holomorphic functions. Bourdon and Shapiro [BoSh90], [Sha93], [BoSh97] have undertaken a thorough investigation of the hypercyclicity of the operator $C_{\varphi}: H^{2} \rightarrow H^{2}, C_{\varphi}(f)=f \circ \varphi$ induced by holomorphic self-maps $\varphi$ of the unit disk $\mathbb{D}$. Their main results are summarised in Tables I and II of [BoSh97]. See also Pavone [Pav92]. Chan and Shapiro [ChSh91] study the hypercyclicity of Birkhoff's translation operator on some Hilbert spaces of entire functions. The existence of closed infinite-dimensional hypercyclic vector manifolds for these operators is investigated by León and Montes [Mon96b], [LeMo97], [Mon98a], [LeMo99']; see also [BeMo95b] and [Mon97].

4b. Holomorphic monsters. Birkhoff's theorem can be interpreted as providing an entire function with wild behaviour near the boundary point $\infty$. A Blaschke product with wild behaviour at every boundary point of the unit disk was constructed by Heins [Hei55]. In a series of papers [Luh78], [Luh79a], [Luh79b], [Luh88] Luh has studied this problem in more general open sets. In [Luh88] he constructed holomorphic functions with extremely wild boundary behaviour in arbitrary simply connected open sets.

Theorem 16 (Luh). Let $O \subset \mathbb{C}, O \neq \mathbb{C}$, be an open set with simply connected components. Then there exists a function $f \in H(O)$ with the following properties:

(i) For every boundary point $\zeta \in \partial O$, for every compact subset $K$ with connected complement and every continuous function $g$ on $K$ that is holomorphic in the interior of $K$, there exist linear transformations $\tau_{n}(z)=a_{n} z+b_{n}$ with $\tau_{n}(K) \subset O(n \in \mathbb{N})$ and $\operatorname{dist}\left(\tau_{n}(K), \zeta\right) \rightarrow 0$ such that $f\left(\tau_{n}(z)\right) \rightarrow g(z)$ uniformly on $K$.

(ii) In addition, each derivative $f^{(j)}(j \in \mathbb{N})$ of $f$ and each anti-derivative of $f$ of arbitrary order has the boundary behaviour described in (i).

Luh calls such functions holomorphic monsters. The author [Gro87] showed that the set of monsters is residual in $H(O)$ and also obtained some kinds of monsters in arbitrary open sets. Monsters with additional universality and other properties were constructed by Luh [Luh97] and Schneider [Scn97]; see also [LMM99+]. Kanatnikov [Kan80], [Kan84] studies universal boundary behaviour (only of $f$ itself) for meromorphic functions. 
4c. Hypercyclic differential operators. In 1952, MacLane [Mac52] showed that there exists an entire function $f$ whose derivatives $f^{(n)}\left(n \in \mathbb{N}_{0}\right)$ form a dense set in the space $H(\mathbb{C})$ of entire functions, in other words, that the differentiation operator $D$ is hypercyclic on $H(\mathbb{C})$. This result was rederived by Blair and Rubel [BlRu83]. Duyos-Ruiz [Duy84] showed the residuality of the set of entire functions that are hypercyclic for $D$; see also [GeSh87] and [Gro87, 2.2.8].

Several authors have constructed $D$-hypercyclic functions on $\mathbb{C}$ or open subsets of $\mathbb{C}$ with additional properties. The least-possible rate of growth was obtained, independently, by the author [Gro90] and Shkarin [Shk93], thus improving on earlier results in [Mac52], [Duy84] and [Hzg88] (see also [Arm99+]. Zero-free hypercyclic functions are obtained by Herzog [Hzg94] and Bernal [Ber97]); functions with additional universality properties are constructed by Blair and Rubel [BlRu84], DuyosRuiz [Duy84], Luh [Luh96], [Luh97] and Schneider [Scn97], where the latter in addition requires that the function be univalent.

The most remarkable generalisation of MacLane's theorem, which at the same time also includes Birkhoff's theorem as a special case, is due to Godefroy and Shapiro [God89], [GoSh91, 5.1]. We fix $N \in \mathbb{N}$ and denote by $T_{a}: \mathbb{C}^{N} \rightarrow \mathbb{C}^{N}$ the translation operator $T_{a} f(z)=f(z+a)$ for $a \in \mathbb{C}^{N}$ and by $D_{k}: \mathbb{C}^{N} \rightarrow \mathbb{C}^{N}$ the differentiation operator $D_{k} f(z)=\frac{\partial f}{\partial z_{k}}(z)$ for $1 \leq k \leq N$.

Theorem 17 (Godefroy, Shapiro). Let $T$ be a continuous linear operator on $H\left(\mathbb{C}^{N}\right)$ that commutes with all translation operators $T_{a}, a \in \mathbb{C}^{N}$ (or, equivalently, with all differentiation operators $\left.D_{k}, 1 \leq k \leq N\right)$. If $T$ is not a scalar multiple of the identity, then $T$ is hypercyclic.

The authors also identify the operators $T$ that satisfy their hypotheses. They are exactly those of the form

$$
T=\Phi(D)=\sum_{|\nu| \geq 0} a_{\nu} D^{\nu}
$$

where $\Phi(z)=\sum_{|\nu| \geq 0} a_{\nu} z^{\nu}$ is an entire function on $\mathbb{C}^{N}$ of exponential type; see [GoSh91, 5.3] for details. Bernal [Ber99b], [Ber99+], cf. [Ber97], studies the universality of sequences $\left(\Phi_{n}(D)\right)$ on arbitrary Runge domains in $\mathbb{C}^{N}$ and the existence of zero-free universal functions, and Aron and Bès [ArBè99] generalise the theorem of Godefroy and Shapiro to a space of entire functions on Banach spaces.

Further hypercyclicity and universality results for differential and related operators are obtained by Mathew [Mth94], Bernal [Ber94], [Ber96] and Bès and Peris $\left[\right.$ Per99 $\left.{ }^{+}\right],\left[B e ̀ P e 99^{+}, 4.4\right]$ for spaces $H(O), O \subset \mathbb{C}$ open; by Chan and Shapiro [ChSh91, Section 4] and León and Montes [LeMo97], [LeMo99 ${ }^{+}$] for Hilbert spaces of entire functions; and by Bonet $\left[\right.$ Bon99 $\left.{ }^{+}\right]$for weighted inductive limits of spaces of holomorphic functions.

4d. Universal power and Taylor series. The first universal power series in the complex plane was constructed by Seleznev [Sel51] in 1951 (but see also [Maz37]). It has radius of convergence 0 with universal approximation properties in $\mathbb{C} \backslash\{0\}$. Corresponding universal power series of radius of convergence 1, hence Taylor series, were obtained by Luh [Luh70], [Luh74a], [Luh76] and Chui and Parnes [ChPa71], where Luh even allowed summability instead of convergence; see also [SMV77], [SeDo77]. 
Theorem 18 (Seleznev, Luh, Chui, Parnes). Let $0 \leq r<\infty$. Then there exists a power series $\sum_{n=0}^{\infty} a_{n} z^{n}$ of radius of convergence $r$ such that for every compact set $K$ in $|z|>r$ with connected complement and any continuous function $f$ on $K$ that is holomorphic in the interior of $K$ there exists an increasing sequence $\left(n_{k}\right)$ of positive integers such that

$$
\sum_{n=0}^{n_{k}} a_{n} z^{n} \rightarrow f(z) \quad \text { uniformly on } K .
$$

This phenomenon is also referred to as universal overconvergence of the power series. Tomm and Trautner [ToTr82] have shown that a universal Taylor series can be found that converges absolutely in $|z| \leq r$; see also [MNP97, §3]. Universal Taylor series on arbitrary simply connected open sets were constructed by Luh [Luh86, Luh97]. Gehlen, Luh and Müller [GeLM99+] have studied properties of such universal Taylor series; in particular, they have shown that these series never have a bounded non-simply connected domain as domain of holomorphy. Residuality results are given in [Gro87, pp. 47-49] and [MNP97, §3]. Luh [Luh97] and Schneider [Scn97] study universal Taylor series with additional universality properties, where the latter in addition requires that the function represented by the power series be univalent.

Recently, Nestoridis [Nes96] obtained universal Taylor series with the stronger property that the compact sets $K$ are allowed to meet the boundary of the disk - that is, the $K$ may be taken from $|z| \geq r$ (necessarily $r>0$ ) - and he showed that this gives a generic universality; see also Kahane [Kah97], [Kah99+]. Subsequently, Nestoridis and Melas $\left[\mathrm{Nes} 99^{+}\right],\left[\mathrm{MeNe} 99^{+}\right]$studied, more generally, corresponding universal Taylor series on arbitrary simply connected open sets and also replaced convergence by summability. The mentioned authors, together with Papadoperakis, Katsoprinakis and Papadimitrakis [Nes96], [MNP97], [KaPa99+], $\left[\mathrm{Nes} 9^{+}\right],\left[\mathrm{MeNe} 99^{+}\right]$, have studied properties of universal Taylor series in the sense of Nestoridis: for example, the growth of coefficients, (non-)summability on the boundary, or additional universality properties.

Results corresponding to Theorem 18 for Dirichlet series, Faber series, Jacobi series and more general series are obtained by Chashchina [Chs63] and Seleznev, Dodunova and co-workers [SMV77], [SeDo77], [SeDo82], [Dod88], [Dod90], [Dod97].

Universal series of simple fractions on the unit circle or on subsets of $\mathbb{C}$ of analytic capacity zero are constructed by Khavinson [Kha61a], [Kha61b].

4e. Universal matrices. The geometric series $\sum_{n=0}^{\infty} z^{n}$ is certainly not universal in the sense of the previous section, but its transforms under a suitable summability matrix may show some universality behaviour. This was first noted by Luh [Luh74b, Satz 7.3] in 1974. Faulstich [Fau79, Satz 5.7] found a universal matrix of weighted mean type. The first regular universal matrix was constructed by Faulstich, Luh and Tomm [FLT81], where a matrix is called regular if it preserves convergence and limits of sequences. For a matrix $A=\left(a_{n k}\right)$ we denote by $\sigma_{n}$ the transforms $\sigma_{n}(z)=\sum_{k=0}^{\infty} a_{n k} \sum_{\nu=0}^{k} z^{\nu}\left(n \in \mathbb{N}_{0}\right)$ of the geometric series provided that they exist.

Theorem 19 (Faulstich, Luh, Tomm). Let $G$ be a simply connected domain with $\mathbb{D} \subset G$ and $1 \notin G$. Then there exists a regular matrix $A=\left(a_{n k}\right)$ with the following properties: 
(i) We have

$$
\sigma_{n}(z) \rightarrow \frac{1}{1-z} \quad \text { locally uniformly in } G ;
$$

(ii) for every compact set $K$ in $\mathbb{C} \backslash \bar{G}$ with connected complement and every continuous function $f$ on $K$ that is holomorphic in the interior of $K$ there exists an increasing sequence $\left(n_{k}\right)$ of positive integers with

$$
\sigma_{n_{k}}(z) \rightarrow f(z) \quad \text { uniformly on } K .
$$

In fact, the matrix $A$ can again be of weighted mean type. Thorpe and Tomm [ThTo85] replace the geometric series by more general series, while the author [Gro92] obtained various residuality results.

\section{Hypercyclic operators in Classical BanaCh SPACES}

Rolewicz [Rol69] was the first to study hypercyclicity of operators in classical Banach spaces. He showed that if $B$ denotes the backward shift

$$
B\left(x_{n}\right)=\left(x_{n+1}\right),
$$

then for any $a>1$ the operator $T=a B$ is hypercyclic on $l^{p}(1 \leq p<\infty)$ and $c_{0}$, and for any $a>0$ it is hypercyclic on the space $\omega$ of all scalar sequences. Since then, shift operators and their generalisations have remained a main source of examples of hypercyclic operators. Recently, Salas [Sal95] succeeded in completely characterising the hypercyclic weighted shift operators; see also $\left[\mathrm{Sal99}^{+}\right]$. On $l^{p}$ $(1 \leq p<\infty)$ or $c_{0}$ a (unilateral) weighted backward shift is an operator

$$
T\left(x_{n}\right)_{n \in \mathbb{N}}=\left(a_{n+1} x_{n+1}\right)_{n \in \mathbb{N}}
$$

with a bounded sequence $\left(a_{n}\right)$ of non-vanishing scalars; on $l^{p}(\mathbb{Z})(1 \leq p<\infty)$ or $c_{0}(\mathbb{Z})$ a bilateral weighted backward shift is an operator

$$
T\left(x_{n}\right)_{n \in \mathbb{Z}}=\left(a_{n+1} x_{n+1}\right)_{n \in \mathbb{Z}} ;
$$

the weighted forward shifts are defined analogously (with $x_{0}=0$ in the unilateral case). We can assume without loss of generality that the $a_{n}$ are positive numbers.

Theorem 20 (Salas).

(a) Let $T$ be a weighted backward shift on $l^{p}(1 \leq p<\infty)$ or $c_{0}$. Then $T$ is hypercyclic if and only if there is an increasing sequence $\left(n_{k}\right)$ of positive integers with

$$
\prod_{n=1}^{n_{k}} a_{n} \rightarrow \infty
$$

(b) Let $T$ be a bilateral weighted backward shift on $l^{p}(\mathbb{Z})(1 \leq p<\infty)$ or $c_{0}(\mathbb{Z})$. Then $T$ is hypercyclic if and only if there is an increasing sequence $\left(n_{k}\right)$ of positive integers such that for all $j \in \mathbb{Z}$

$$
\prod_{n=1}^{n_{k}} a_{j+n} \rightarrow \infty \quad \text { and } \quad \prod_{n=1}^{n_{k}} a_{j-n} \rightarrow 0 .
$$

We remark that Salas' original proof for $l^{2}$ also works for the other spaces $l^{p}$ and for $c_{0}$; cf. [Sal95, p. 1003]. By symmetry, the corresponding result for bilateral weighted forward shifts is obtained on interchanging the limits $\infty$ and 0 , while no unilateral weighted forward shift can be hypercyclic; see [Sal95]. 
Salas' theorem covers several previous results on shift operators; see [Rol69], [Kit82, 1.8, 1.10], [GeSh87, Section 4], [Bea88, III.C] and [Sal91]. Bès and Peris [BèPe99 ${ }^{+}$, Sections 3 and 4] characterise hereditary hypercyclicity (with respect to given subsequences) of weighted shift operators on $l^{2}$ and study the corresponding sets of universal elements. Hypercyclicity of certain weighted shifts on some specific Fréchet spaces are studied by Mathew [Mth94] and Gulisashvili and MacCluer [GuMa96]. Martínez and Peris [MaPe99 $\left.{ }^{+}\right]$have extended Salas' result to Köthe echelon spaces, while the extension to arbitrary Fréchet sequence spaces in which the canonical unit vectors $e_{n}$ form a Schauder basis is given in [Gro99 ${ }^{+}$.

Rolewicz [Rol69] also studied the analogues of his result for the continuous semigroup of translations on function spaces. Recently, Desch, Schappacher and Webb [DSW97, §4] obtained the corresponding analogues of Salas' theorem.

The hypercyclicity of further operators in a Banach or Hilbert space setting was studied by Herrero and Wang [HeWa90] and Chan and Shapiro [ChSh91, Sections $3,4]$ (compact perturbations of the identity and of other operators); Godefroy and Shapiro [GoSh91, Sections 3, 4], Ansari [Ans95, Theorem 5], and Bès, Martínez and Peris [BèPe99 $\left.{ }^{+}, 2.11\right],\left[\mathrm{MaPe} 99^{+}\right]$(generalised backward shift operators and related operators, adjoints of multiplication operators); Protopopescu and Azmy [PrAz92] and MacCluer [Mcc92], see also [DSW97] (exponentials of shift and related operators); Protopopescu and Azmy [PrAz92], Salas [Sal95, Section 3], [Sal99+, Section 6], and Bès, Martínez and Peris [BèPe99 $\left.{ }^{+}, 2.9\right]$, [MaPe99 ${ }^{+}$] (perturbations of the identity by shift or generalised backward shift operators). For hypercyclic operators in Banach or Hilbert spaces of holomorphic functions we refer to Sections $4 \mathrm{a}$ and $4 \mathrm{c}$ of this paper.

León and Montes [Mon96b], [LeMo97], [LeMo99 $\left.{ }^{+}\right]$have investigated many of the operators mentioned above regarding the existence of infinite-dimensional closed hypercyclic vector manifolds. In particular, it turned out that Rolewicz' operator $T=a B(a>1)$ on $l^{p}$ possesses no such manifolds; see [Mon96b, 3.4], [Mon97].

Recently, Chan [Cha99] defined a notion of hypercyclicity for operators on the algebra $B(H)$ of bounded linear operators on a Hilbert space $H$. In particular, he relates the hypercyclicity of an operator $T$ on $H$ to the hypercyclicity, in the strong operator topology, of the induced operator $L_{T}(V)=T V$ on $B(H)$.

\section{A CONCRETE universal object: the Riemann Zeta-FunCtion}

All the universalities (and hypercyclicities) that have been studied in the literature share one feature: A corresponding universal object is either constructed in some countable inductive process or its existence is guaranteed by an application of the Baire category theorem. But in no case could a universal object be written down explicitly (a statement that is admittedly rather vague) - with one exception. In 1975 Voronin [Vor75a], [Vor75b] obtained the remarkable result that the Riemann zeta-function enjoys some kind of translation universality à la Birkhoff. Let $K_{r}=K_{r}\left(\frac{3}{4}\right)$ denote the closed disk of radius $r, 0<r<\frac{1}{4}$, centred at $z=\frac{3}{4}$; that is, $K_{r}$ lies inside $\frac{1}{2}<\operatorname{Re} z<1$, the right half of the critical strip.

Theorem 21 (Voronin). For any $r, 0<r<\frac{1}{4}$, any continuous function $f$ on $K_{r}$ that is holomorphic and zero-free in the interior of $K_{r}$, and any $\varepsilon>0$ there exists some $t>0$ such that

$$
\max _{z \in K_{r}}|\zeta(z+i t)-f(z)|<\varepsilon
$$


We remark that approximability of a single function $f$ with an isolated zero would contradict the Riemann hypothesis [Rei80, p. 450]. In the sequel the result was strengthened so that in fact for any compact set $K$ in $\frac{1}{2}<\operatorname{Re} z<1$ with connected complement and any function $f$ on $K$ as above we have

$$
\underline{d}\left(\left\{t>0: \max _{z \in K}|\zeta(z+i t)-f(z)|<\varepsilon\right\}\right)>0,
$$

where $\underline{d}$ denotes lower density (Reich [Rei77], Bagchi [Bag81], [Bag82]; see also Laurinčikas [Lau95]). Also, the $t$ may be chosen from any fixed arithmetic progression $(m \Delta)_{m \in \mathbb{N}}, \Delta>0$, with lower density then calculated in $\mathbb{N}$ (Reich [Rei80], [Rei82]).

Corresponding results for Dedekind zeta-functions, for all Dirichlet $L$-functions and other Dirichlet series, for Hurwitz and Lerch zeta-functions, for certain Euler products and other related functions were obtained by Voronin [Vor75a], [Vor75b], [Vor77], [Vor84], Reich [Rei77], [Rei80], [Rei82], Gonek [Gon79], Laurinčikas and coworkers [Lau79a], [Lau79b], [Lau82], [Lau83], [Lau84], [Lau97], [Gar97], [Lau98a], [Lau98b], [KaLa98], [LaMa99+] and Bagchi [Bag81, Bag82]; see also [Goo81] and [Èmi90].

Some functions were also found where the requirement that $f$ be zero-free can be dropped; examples are the derivative $\zeta^{\prime}$ of the Riemann zeta-function, $\log \zeta$, Hurwitz and Lerch zeta-functions and others; see Gonek [Gon79], Bagchi [Bag81], [Bag82, 3.4], Gavrilov and Kanatnikov [GaKa82], Voronin [Vor77], [KaVo92, Chapter VII, §1] and Laurinčikas and Garunkštis [Lau97], [Gar97], [Lau98a].

For treatments of these topics in textbooks we refer to [KaVo92, Chapter VII] and [Lau96, Sections 6.5 and 9.2].

\section{REFERENCES}

[1] W. H. Gottschalk and G. A. Hedlund: Topological dynamics, Amer. Math. Soc., Providence, RI, 1955. MR 17:650e

[2] H. Jarchow: Locally convex spaces, B. G. Teubner, Stuttgart, 1981. MR 83h:46008

[3] J. C. Oxtoby: Measure and category, Second edition, Springer, New York, 1980. MR 81j:28003

[4] H. Toruńczyk: Characterizing Hilbert space topology, Fund. Math. 111 (1981), 247-262. MR 82i: 57016

[5] P. Walters: An introduction to ergodic theory, Springer, New York, 1982. MR 84e:28017

\section{BIBLIOGRAPHY}

At the end of each entry we refer to the section(s) in which the work is cited.

[Abe97] Y. Abe, Universal holomorphic functions in several variables, Analysis 17 (1997), 71-77. MR 98e:32003

[Abe99 $\left.{ }^{+}\right] \quad$ Y. Abe, Universal functions on complex special linear groups, (Proc. Conf., Poznań, 1998) (to appear).

[AbZa99+] Y. Abe and P. Zappa, Universal functions on complex general linear groups, J. Approx. Theory (to appear).

[AlAr98a] M. P. Aldred and D. H. Armitage, Harmonic analogues of G. R. MacLane's universal functions, J. London Math. Soc. (2) 57 (1998), 148-156. MR 99g:31006 3e

[AlAr98b] M. P. Aldred and D. H. Armitage, Harmonic analogues of G. R. MacLane's universal functions. II, J. Math. Anal. Appl. 220 (1998), 382-395. MR 99g:31007 3e

[Ale60] G. Alexits, Konvergenzprobleme der Orthogonalreihen, Verlag Ungar. Akad. Wiss., Budapest, 1960. English edition: Convergence problems of orthogonal series, Pergamon Press, Oxford, 1961. MR 28:5292

[Ans95] S. I. Ansari, Hypercyclic and cyclic vectors, J. Funct. Anal. 128 (1995), 374-383. MR 96h: 47002 
[Ans97] S. I. Ansari, Existence of hypercyclic operators on topological vector spaces, J. Funct. Anal. 148 (1997), 384-390. MR 98h:47028a

$2 \mathrm{~b}, 2 \mathrm{c}, 2 \mathrm{~d}$

[Arm99+] D. H. Armitage, Dense vector spaces of universal harmonic functions, Advances in multivariate approximation (Proc. Conf., Witten-Bommerholz, 1998) (to appear).

3e, $4 \mathrm{c}$

[ArGa96] D. H. Armitage and P. M. Gauthier, Recent developments in harmonic approximation, with applications, Results Math. 29 (1996), 1-15. MR 97f:31007 3e

[ArBè99] R. Aron and J. Bès, Hypercyclic differentiation operators, Function Spaces (Proc. Conf., Edwardsville, IL, 1998), 39-46, Amer. Math. Soc., Providence, RI, 1999. 4a,4c

[AvCa83] V. Aversa and R. Carrese, Una primitiva universale per funzioni di piu variabili, Rend. Circ. Mat. Palermo (2) 32 (1983), 131-138. MR 85b:26012 3b

[Bag81] B. Bagchi, The statistical behaviour and universality properties of the Riemann zeta function and other allied Dirichlet series, Thesis, Indian Statistical Institute, Calcutta, 1981.

[Bag82] B. Bagchi, A joint universality theorem for Dirichlet L-functions, Math. Z. 181 (1982), 319-334. MR 84c:10038

[BaKr81] A. V. Bakhshetsyan and V. G. Krotov, On universal series in Schauder systems (Russian), Izv. Akad. Nauk Armyan. SSR Ser. Mat. 16 (1981), 44-53. English transl. in: Soviet J. Contemporary Math. Anal. 16 (1981), no. 1, 33-41. MR 84d:42035 3c

[Bar61] N. K. Bari, Trigonometric series (Russian), Fizmatgiz, Moscow, 1961. English transl.: N. K. Bary, A treatise on trigonometric series. Vols. I, II, Pergamon Press, Oxford, 1964. MR 23:A3411

[Bea86] B. Beauzamy, Un opérateur, sur l'espace de Hilbert, dont tous les polynômes sont hypercycliques, C. R. Acad. Sci. Paris Sér. I Math. 303 (1986), 923-925. MR 88g:47010

$2 \mathrm{a}, \mathbf{2 b}$

[Bea87a] B. Beauzamy, An operator on a separable Hilbert space with many hypercyclic vectors, Studia Math. 87 (1987), 71-78. MR 89j:47004

$\mathbf{2 a}, \mathbf{2 b}$

[Bea87b] B. Beauzamy, Opérateurs de rayon spectral strictement supérieur à 1, C. R. Acad. Sci. Paris Sér. I Math. 304 (1987), 263-266. MR 88d:47004 2a,2d

[Bea88] B. Beauzamy, Introduction to operator theory and invariant subspaces, NorthHolland, Amsterdam, 1988. MR 90d:47001 1a,1b,2a,2b,2c,2d,5

[Bea90] B. Beauzamy, An operator on a separable Hilbert space with all polynomials hypercyclic, Studia Math. 96 (1990), 81-90. MR 91d:47004

[Ber94] L. Bernal-González, Derivative and antiderivative operators and the size of complex domains, Ann. Polon. Math. 59 (1994), 267-274. MR 95k:30081 1c,2a,4c

[Ber96] L. Bernal-González, Universal functions for Taylor shifts, Complex Variables Theory Appl. 31 (1996), 121-129. MR 98h:47042 1c,2a,4c

[Ber97] L. Bernal-González, On universal entire functions with zero-free derivatives, Arch. Math. 68 (1997), 145-150. MR 97k:30048

[Ber99a] L. Bernal-González, On hypercyclic operators on Banach spaces, Proc. Amer. Math. Soc. 127 (1999), 1003-1010. MR 99f:47010

[Ber99b] L. Bernal-González, Hypercyclic sequences of differential and antidifferential operators, J. Approx. Theory 96 (1999), 323-337. CMP 99:08 1c,2a,4c

[Ber99 ${ }^{+}$L. Bernal-González, Densely hereditarily hypercyclic sequences and large hypercyclic mainfolds, Proc. Amer. Math. Soc. (to appear). CMP 99:02 1a,1b,1c,2a,2c,4c

[BeCa99+ ${ }^{+}$L. Bernal-González and M. C. Calderón-Moreno, A Seidel-Walsh theorem with linear differential operators, Arch. Math. (to appear).

[BeMo95a] L. Bernal-González and A. Montes-Rodríguez, Universal functions for composition operators, Complex Variables Theory Appl. 27 (1995), 47-56. MR 96a:30041 4a

[BeMo95b] L. Bernal González and A. Montes Rodríguez, Non-finite dimensional closed vector spaces of universal functions for composition operators, J. Approx. Theory 82 (1995), 375-391. MR 96f:30034 2b,4a

$\left[\right.$ Brn99 $\left.{ }^{+}\right] \quad$ N. C. Bernardes, On orbits of polynomial maps in Banach spaces, Quaestiones Math. (to appear).

[Bès99] J. P. Bès, Invariant manifolds of hypercyclic vectors for the real scalar case, Proc. Amer. Math. Soc. 127 (1999), 1801-1804. CMP 99:10

$\left[\right.$ BèPe99 $\left.{ }^{+}\right]$J. Bès and A. Peris, Hereditarily hypercyclic operators, J. Funct. Anal. (to appear). 
[Bir29] G. D. Birkhoff, Démonstration d'un théorème élémentaire sur les fonctions entières, C. R. Acad. Sci. Paris 189 (1929), 473-475. JFM 55:192 1a,2a,4a

[BlRu83] C. Blair and L. A. Rubel, A universal entire function, Amer. Math. Monthly 90 (1983), 331-332. MR 85a:30046 4c

[BlRu84] C. Blair and L. Rubel, A triply universal entire function, Enseign. Math. (2) 30 (1984), 269-274. MR 86b:30034 1b,4a,4c

[Bog78] A. Bogmér, On universal elements of series of linear operators (Hungarian), Mat. Lapok 31 (1978/83), 195-196. MR 85h:41049

[BoSö87] A. Bogmér and A. Sövegjártó, On universal functions, Acta Math. Hungar. 49 (1987), 237-239. MR 88a:26006 3b

$\left[\right.$ Bon99 $\left.{ }^{+}\right] \quad$ J. Bonet, Hypercyclic and chaotic convolution operators, J. London Math. Soc. (to appear). $\quad 2 c, 2 d, 3 e, 4 c$

[BoPe98] J. Bonet and A. Peris, Hypercyclic operators on non-normable Fréchet spaces, J. Funct. Anal. 159 (1998), 587-595. CMP 99:04 2d

[Bou93] P. S. Bourdon, Invariant manifolds of hypercyclic vectors, Proc. Amer. Math. Soc. 118 (1993), 845-847. MR 93i:47002 2b

[Bou96] P. S. Bourdon, The second iterate of a map with dense orbit, Proc. Amer. Math. Soc. 124 (1996), 1577-1581. MR 97a:54043

[BoSh90] P. S. Bourdon and J. H. Shapiro, Cyclic composition operators on $H^{2}$, Operator Theory: Operator Algebras and Applications, Part 2 (Proc. Summer Res. Inst., Durham, NH, 1988), 43-53, Amer. Math. Soc., Providence, RI, 1990. MR 91h:47028 1c,4a

[BoSh97] P. S. Bourdon and J. H. Shapiro, Cyclic phenomena for composition operators, Mem. Amer. Math. Soc. 125 (1997), no. 596. MR 97h:47023 2c,4a

[Bru78] A. M. Bruckner, Differentiation of real functions, Springer, Berlin, 1978. MR 80h:26002

3b

[Buc87] Z. Buczolich, On universal functions and series, Acta Math. Hungar. 49 (1987), 403-414. MR 88k:42011 3b,3c

[CaWa98] X. H. Cao and Y. G. Wang, Hypercyclic and supercyclic operators (Chinese), Qufu Shifan Daxue Xuebao Ziran Kexue Ban 24 (1998), no. 4, 4-7. CMP 99:08 2d

[Cat89] F. S. Cater, Some higher dimensional Marcinkiewicz theorems, Real Anal. Exchange 15 (1989/90), 269-274. MR 91d:26008 3b

[Cha99] K. C. Chan, Hypercyclicity of the operator algebra for a separable Hilbert space, J. Operator Theory 42 (1999), 231-244. 2 2b,5

[Cha99 $\left.{ }^{+}\right] \quad$ K. C. Chan, The density of hypercyclic operators on a Hilbert space, preprint. 2d

[ChSh91] K. C. Chan and J. H. Shapiro, The cyclic behavior of translation operators on Hilbert spaces of entire functions, Indiana Univ. Math. J. 40 (1991), 1421-1449. MR 92m:47060 2c,2d,4a,4c,5

[Chs63] N. S. Chashchina, On the theory of a universal Dirichlet series (Russian), Izv. Vyssh. Uchebn. Zaved. Mat. 1963, no. 4(35), 165-167. MR 27:3787 4d

[Che79] P. S. Chee, Universal functions in several complex variables, J. Austral. Math. Soc. Ser. A 28 (1979), 189-196. MR 81c:32014 4a

[Chk77] G. A. Chkhaidze, Universal series (Russian), Soobshch. Akad. Nauk Gruzin. SSR 86 (1977), 45-47. MR 57:3731

[ChPa71] C. K. Chui and M. N. Parnes, Approximation by overconvergence of a power series, J. Math. Anal. Appl. 36 (1971), 693-696. MR 45:563

$4 d$

[DSW97] W. Desch, W. Schappacher, and G. F. Webb, Hypercyclic and chaotic semigroups of linear operators, Ergodic Theory Dynam. Systems 17 (1997), 793-819. MR 98j: 47083

$2 \mathrm{a}, 2 \mathrm{c}, 2 \mathrm{~d}, 3 \mathrm{e}, 5$

[Dod88] L. K. Dodunova, On the overconvergence of universal series (Russian), Izv. Vyssh. Uchebn. Zaved. Mat. 1988, no. 2, 19-22. English transl. in: Soviet Math. (Iz. VUZ) 32 (1988), no. 2, 27-30. MR 89d:30003

$4 d$

[Dod90] L. K. Dodunova, A generalization of the universality property of Faber polynomial series (Russian), Izv. Vyssh. Uchebn. Zaved. Mat. 1990, no. 12, 31-34. English transl. in: Soviet Math. (Iz. VUZ) 34 (1990), no. 12, 37-40. MR 92i:30004 4d

[Dod97] L. K. Dodunova, Approximation of analytic functions by de la Vallée-Poussin sums (Russian), Izv. Vyssh. Uchebn. Zaved. Mat. 1997, no. 3, 34-37. English transl. in: Russian Math. (Iz. VUZ) 41 (1997), no. 3, 33-36. MR 99g:41040 
[Duy83] S. M. Duו̆os Ruis [S. M. Duyos-Ruiz], On the existence of universal functions (Russian), Dokl. Akad. Nauk SSSR 268 (1983), 18-22. English transl. in: Soviet Math. Dokl. 27 (1983), 9-13. MR 84d:30047 $3 \mathbf{e}, 4 \mathbf{a}$

[Duy84] S. M. Duı̆os Ruis [S. M. Duyos-Ruiz], Universal functions and the structure of the space of entire functions (Russian), Dokl. Akad. Nauk SSSR 279 (1984), 792795. English transl. in: Soviet Math. Dokl. 30 (1984), 713-716. MR 86c:30055

$1 \mathrm{a}, \mathbf{1 b}, \mathbf{4 a}, \mathbf{4 c}$

[DVW97] J. Dyson, R. Villella-Bressan, and G. Webb, Hypercyclicity of solutions of a transport equation with delays, Nonlinear Anal. 29 (1997), 1343-1351. MR 98k:35201 2a,3e

[Dza64] O. P. Dzagnidze, On universal double series (Russian), Soobshch. Akad. Nauk Gruzin. SSR 34 (1964), 525-528. MR 29:2566

[Dza69] O. P. Dzagnidze, The universal harmonic function in the space $E_{n}$ (Russian), Soobshch. Akad. Nauk Gruzin. SSR 55 (1969), 41-44. MR 41:5640 3e

[Edg70] J. J. Edge, Universal trigonometric series, J. Math. Anal. Appl. 29 (1970), 507-511. MR 40:4654

[Ema97] H. Emamirad, Hypercyclicité du semi-groupe de transport et le théorème de représentation de Lax et Phillips, C. R. Acad. Sci. Paris Sér. I Math. 325 (1997), 157-162. MR 98h:47058

$\mathbf{2 a}, \mathbf{3 e}$

[Ema98] H. Emamirad, Hypercyclicity in the scattering theory for linear transport equation, Trans. Amer. Math. Soc. 350 (1998), 3707-3716. MR 98k:47077 2a,3e

[Ėmi90] K. M. Èminyan, $\chi$-universality of the Dirichlet L-function (Russian), Mat. Zametki 47 (1990), no. 6, 132-137. English transl. in: Math. Notes 47 (1990), 618-622. MR 91i:11103

[Fau79] K. Faulstich, Summierbarkeit von Potenzreihen durch Riesz-Verfahren mit komplexen Erzeugendenfolgen, Mitt. Math. Sem. Giessen 139 (1979). MR 80j:40002

[FLT81] K. Faulstich, W. Luh, and L. Tomm, Universelle Approximation durch RieszTransformierte der geometrischen Reihe, Manuscripta Math. 36 (1981), 309-321. MR 84a: 40005

[Fly95] E. Flytzanis, Unimodular eigenvalues and linear chaos in Hilbert spaces, Geom. Funct. Anal. 5 (1995), 1-13. MR 95k:28034 2a,2d

[GaSt94] X.-X. Gan and K. R. Stromberg, On universal primitive functions, Proc. Amer. Math. Soc. 121 (1994), 151-161. MR 94g:26024

[Gar97] R. Garunkštis, The universality theorem with weight for the Lerch zeta-function, New Trends in Probability and Statistics, Vol. 4 (Proc. Conf., Palanga, 1996), 59-67, VSP, Utrecht; TEV, Vilnius, 1997. CMP 99:03

[Gau94] P. M. Gauthier, Uniform approximation, Complex Potential Theory (Proc. NATO Adv. Study Inst., Montreal, 1993), 235-271, Kluwer Acad. Publ., Dordrecht, 1994. MR 96d:30048

[GaKa82] V. I. Gavrilov and A. N. Kanatnikov, An example of a universal holomorphic function (Russian), Dokl. Akad. Nauk SSSR 265 (1982), 274-276. English transl. in: Soviet Math. Dokl. 26 (1982), 52-54. MR 83j:10047

$\left[\right.$ GeLM99 $\left.^{+}\right]$W. Gehlen, W. Luh, and J. Müller, On the existence of O-universal functions, Complex Variables Theory Appl. (to appear).

[GeOl64] B. R. Gelbaum and J. M. H. Olmsted, Counterexamples in analysis, Holden-Day, San Francisco, CA, 1964. MR 30:204

[GeSh87] R. M. Gethner and J. H. Shapiro, Universal vectors for operators on spaces of holomorphic functions, Proc. Amer. Math. Soc. 100 (1987), 281-288. MR 88g:47060

1a,1b,1c,2a,4a, 4c,5

[God89] G. Godefroy, Opérateurs ayant un sous-espace dense de vecteurs hypercycliques, Séminaire d'Initiation à l'Analyse 1988-1989 (1989), Exposé no. 3. MR 92d:47028

$1 \mathrm{c}, 2 \mathrm{a}, 2 \mathrm{~b}, 4 \mathrm{a}, \mathbf{4 c}$

[GoSh91] G. Godefroy and J. H. Shapiro, Operators with dense, invariant, cyclic vector manifolds, J. Funct. Anal. 98 (1991), 229-269. MR 92d:47029

$1 \mathrm{a}, 1 \mathrm{~b}, 1 \mathrm{c}, 2 \mathrm{a}, 2 \mathrm{~b}, 2 \mathrm{c}, 2 \mathrm{~d}, 3 \mathrm{e}, 4 \mathrm{a}, 4 \mathrm{c}, 5$

[GoPe65] C. Goffman and G. Pedrick, First course in functional analysis, Prentice-Hall, Englewood Cliffs, N.J., 1965. MR 32:1540 
[GoWa60] C. Goffman and D. Waterman, Basic sequences in the space of measurable functions, Proc. Amer. Math. Soc. 11 (1960), 211-213. MR 22:2886

1d,3d

[GoWa72] C. Goffman and D. Waterman, A remark concerning universal series, J. Math. Anal. Appl. 40 (1972), 735-737. MR 47:7409 3d

[Gon79] S. M. Gonek, Analytic properties of zeta and L-functions, Thesis, Univ. of Michigan, Ann Arbor, 1979

[GoLM99+] M. González-Ortiz, F. León-Saavedra, and A. Montes-Rodríguez, Semi-Fredholm theory: hypercyclic and supercyclic subspaces, preprint. $\quad \mathbf{2 b}, \mathbf{2 c}, \mathbf{2 d}$

[Goo81] A. Good, On the distribution of the values of Riemann's zeta-function, Acta Arith. 38 (1981), 347-388. MR 83m:10068

[Gra84] E. Grande, Sur un théorème de Marcinkiewicz, Problemy Mat. 1984, no. 4, 35-41. MR 86b:26012

$3 \mathrm{~b}$

[Gro87] K.-G. Grosse-Erdmann, Holomorphe Monster und universelle Funktionen, Mitt. Math. Sem. Giessen 176 (1987). MR 88i:30060 1a,1b,1c,3a,3c,4a,4b,4c,4d

[Gro90] K.-G. Grosse-Erdmann, On the universal functions of G. R. MacLane, Complex Variables Theory Appl. 15 (1990), 193-196. MR 91i:30021 1c,4c

[Gro92] K.-G. Grosse-Erdmann, Topologies on matrix spaces and universal matrices, Analysis 12 (1992), 47-56. MR 93d:46016

$\left[\mathrm{Gro}^{+} 9^{+}\right] \quad$ K.-G. Grosse-Erdmann, Hypercyclic and chaotic weighted shifts, preprint. $\mathbf{5}$

[GuMa96] A. Gulisashvili and C. R. MacCluer, Linear chaos in the unforced quantum harmonic oscillator, J. Dynam. Systems Measurement Control 118 (1996), 337-338. Zbl 870:58057

[HNRR86] D. Hadwin, E. Nordgren, H. Radjavi, and P. Rosenthal, Orbit-reflexive operators, J. London Math. Soc. (2) 34 (1986), 111-119. MR 88d:47010 2b

[HKR85] I. Halperin, C. Kitai, and P. Rosenthal, On orbits of linear operators, J. London Math. Soc. (2) 31 (1985), 561-565. MR 87e:47025 2a,2d

[Hei55] M. Heins, A universal Blaschke product, Arch. Math. 6 (1955), 41-44. MR 16:460e

$4 a, 4 b$

[Hej89] J. Hejduk, Universal sequences in the space of real measurable functions, Zeszyty Nauk. Politech. Łódz. Mat. no. 21 (1989), 75-85. MR 91d:28002

[Her91] D. A. Herrero, Limits of hypercyclic and supercyclic operators, J. Funct. Anal. 99 (1991), 179-190. MR 92g:47026 2a,2b,2c,2d

[Her92] D. A. Herrero, Hypercyclic operators and chaos, J. Operator Theory 28 (1992), 93103. MR 95g: $47031 \quad$ 2c,2d

[HeKi92] D. A. Herrero and C. Kitai, On invertible hypercyclic operators, Proc. Amer. Math. Soc. 116 (1992), 873-875. MR 93a:47023 2c

[HeWa90] D. A. Herrero and Z.-Y. Wang, Compact perturbations of hypercyclic and supercyclic operators, Indiana Univ. Math. J. 39 (1990), 819-829. MR 91k:47042 2d,5

[Hzg88] G. Herzog, Universelle Funktionen, Diplomarbeit, Univ. Karlsruhe, Karlsruhe, 1988.

$3 e, 4 c$

[Hzg91] G. Herzog, On universal functions and interpolation, Analysis 11 (1991), 21-26. MR 93c: 41002

[Hzg94] G. Herzog, On zero-free universal entire functions, Arch. Math. 63 (1994), 329-332. MR 95f:54029 1b,4c

[Hzg95] G. Herzog, On a theorem of Seidel and Walsh, Period. Math. Hungar. 30 (1995), 205-210. MR 96e:30116 1b,4a

[Hzg97] G. Herzog, On a universality of the heat equation, Math. Nachr. 188 (1997), 169-171. MR 98i:35077 3e

[HeLe93] G. Herzog and R. Lemmert, Über Endomorphismen mit dichten Bahnen, Math. Z. 213 (1993), 473-477. MR 94h:47052 2b,2d

[HeLe98] G. Herzog and R. Lemmert, On universal subsets of Banach spaces, Math. Z. 229 (1998), 615-619. CMP 99:06

[HeSc94] G. Herzog and C. Schmoeger, On operators $T$ such that $f(T)$ is hypercyclic, Studia Math. 108 (1994), 209-216. MR 95f:47031 2d

[Hor87] M. Horváth, On multidimensional universal functions, Studia Sci. Math. Hungar. 22 (1987), 75-78. MR 88m:26013 
[Iva81] V. I. Ivanov, Representation of measurable functions by multiple trigonometric series (Russian), Dokl. Akad. Nauk SSSR 259 (1981), 279-282. English transl. in: Soviet Math. Dokl. 24 (1981), 65-68. MR 83b:42016

[Iva83a] V. I. Ivanov, Representation of measurable functions by multiple trigonometric series (Russian), Trudy Mat. Inst. Steklov. 164 (1983), 100-123. English transl. in: Proc. Steklov Inst. Math. 164 (1983), 113-139. MR 86e:42023

[Iva83b] V. I. Ivanov, The coefficients of orthogonal universal series and zero series (Russian), Dokl. Akad. Nauk SSSR 272 (1983), 19-23. English transl. in: Soviet Math. Dokl. 28 (1983), 312-315. MR 85d:42028

3c

[Iva86] V. I. Ivanov, Representation of functions by series in metric symmetric spaces without linear functionals (Russian), Dokl. Akad. Nauk SSSR 289 (1986), 532-535. English transl. in: Soviet Math. Dokl. 34 (1987), 113-116. MR 87j:46058 1d,3d

[Iva89] V. I. Ivanov, Representation of functions by series in metric symmetric spaces without linear functionals (Russian), Trudy Mat. Inst. Steklov. 189 (1989), 34-77. English transl. in: Proc. Steklov Inst. Math. 189 (1990), 37-85. MR 90i:41045 1d,3c,3d

[Joó78] I. Joó, Note to a theorem of Talaljan on universal series and to a problem of Nikišin, Fourier Analysis and Approximation Theory (Proc. Colloq., Budapest, 1976), Vol. I, 451-458, North-Holland, Amsterdam, 1978. MR 81k:42025 1c,3c

[Joó89] I. Joó, On the divergence of eigenfunction expansions, Ann. Univ. Sci. Budapest. Eötvös Sect. Math. 32 (1989), 3-36. MR 92d:47030 1a,3b,3c

[Joó91] I. Joó, On a theorem of Miklós Horváth (Hungarian), Mat. Lapok 34 (1983/87), 301-306 (1991). MR 93h:26018

[KaLa98] A. Kachenas and A. Laurinchikas, On Dirichlet series associated with some parabolic forms (Russian), Liet. Mat. Rink. 38 (1998), 82-97. English transl. in: Lithuanian Math. J. 38 (1998) (to appear). CMP 99:06

[KaSt58] S. Kachmazh [S. Kaczmarz] and G. Shteľngauz [H. Steinhaus], Theory of orthogonal series (Russian), Gosudarstv. Izdat. Fiz.-Mat. Lit., Moscow, 1958. MR 20:1148 3c

[Kah97] J.-P. Kahane, General properties of Taylor series 1896-1996, J. Fourier Anal. Appl. 3 (1997), Special Issue, 907-911. CMP 98:09

$4 d$

$\left[\right.$ Kah99 $\left.^{+}\right]$J.-P. Kahane, Baire theory in Fourier and Taylor series (Proc. Conf., Philadelphia, PA, 1996) (to appear).

$4 d$

[Kan80] A. N. Kanatnikov, Limiting sets along sequences of compacta (Russian), Dokl. Akad. Nauk SSSR 253 (1980), 14-17. English transl. in: Soviet Math. Dokl. 22 (1980), 5-9. MR 82g:30056

[Kan84] A. N. Kanatnikov, Limit sets of meromorphic functions with respect to sequences of compacta (Russian), Izv. Akad. Nauk SSSR Ser. Mat. 48 (1984), 1196-1213. English transl. in: Math. USSR-Izv. 25 (1985), 501-517. MR 86k:30038

$4 \mathrm{~b}$

[KaVo92] A. A. Karatsuba and S. M. Voronin, The Riemann zeta-function, Walter de Gruyter, Berlin, 1992. MR 93h:11096

$\left[\mathrm{KaPa}^{+}\right]$E. S. Katsoprinakis and M. Papadimitrakis, Extensions of a theorem of Marcinkiewicz-Zygmund and of Rogosinski's formula and an application to universal Taylor series, Proc. Amer. Math. Soc. (to appear). CMP 99:01

[Kha61a] S. Ya. Khavinson, Some problems concerning the completeness of systems (Russian), Dokl. Akad. Nauk SSSR 137 (1961), 793-796. English transl. in: Soviet Math. Dokl. 2 (1961), 358-361. MR 23:A1044

$1 \mathrm{~d}, \mathbf{4 d}$

[Kha61b] S. Ya. Khavinson, On approximation, with reference to the size of the coefficients of the approximants (Russian), Trudy Mat. Inst. Steklov. 60 (1961), 304-324. English transl. in: Amer. Math. Soc. Transl. (2) 44 (1965), 67-88. MR 25:183 4d

[Kit82] C. Kitai, Invariant closed sets for linear operators, Thesis, Univ. of Toronto, Toronto, $1982 . \quad$ 1a, 1b,1c,2a,2b,2c,2d,5

[Kör89] T. W. Körner, Universal trigonometric series with rapidly decreasing coefficients, Commutative Harmonic Analysis (Proc. Conf., Canton, NY, 1987), 115-149, Amer. Math. Soc., Providence, RI, 1989. MR 90g:42011 3c

[Koz50] V. Ya. Kozlov, On complete systems of orthogonal functions (Russian), Mat. Sb. (N.S.) 26(68) (1950), 351-364. MR 12:174g

[Kro74] V. G. Krotov, Representation of measurable functions by series in the FaberSchauder system, and universal series (Russian), Dokl. Akad. Nauk SSSR 214 
(1974), 1258-1261. English transl. in: Soviet Math. Dokl. 15 (1974), 351-355. MR 51:1262

[Kro75] V. G. Krotov, Universal Fourier series in the Faber-Schauder system (Russian), Vestnik Moskov. Univ. Ser. I Mat. Mekh. 30 (1975), no. 4, 53-58. English transl. in: Moscow Univ. Math. Bull. 30 (1975), no. 3-4, 101-105. MR 54:5726

[Kro77] V. G. Krotov, Representation of measurable functions by series in the FaberSchauder system, and universal series (Russian), Izv. Akad. Nauk SSSR Ser. Mat. 41 (1977), 215-229. English transl. in: Math. USSR-Izv. 11 (1977), 205-218. MR 55:10954

$3 \mathbf{c}$

[Kro91] V. G. Krotov, On the smoothness of universal Marcinkiewicz functions and universal trigonometric series (Russian), Izv. Vyssh. Uchebn. Zaved. Mat. 1991, no. 8, 26-31. English transl. in: Soviet Math. (Iz. VUZ) 35 (1991), no. 8, 24-28. MR 94a:42002

$3 \mathrm{~b}, 3 \mathrm{c}$

[Lam74] C. W. Lamb, Representation of functions as limits of martingales, Trans. Amer. Math. Soc. 188 (1974), 395-405. MR 49:4087

[Lau79a] A. Laurincikas, Distribution des valeurs de certaines séries de Dirichlet, C. R. Acad. Sci. Paris Sér. A 289 (1979), A43-A45. MR 80g:10038 6

[Lau79b] A. Laurinčikas, Sur les séries de Dirichlet et les polynômes trigonométriques, Séminaire de Théorie des Nombres 1978-1979 (1979), Exposé no. 24. MR 81m:10080

[Lau82] A. Laurinchikas, Distribution of values of generating Dirichlet series of multiplicative functions (Russian), Litovsk. Mat. Sb. 22 (1982), no. 1, 101-111. English transl. in: Lithuanian Math. J. 22 (1982), 56-63. MR 83m:10074

[Lau83] A. Laurinchikas, The universality theorem (Russian), Litovsk. Mat. Sb. 23 (1983), no. 3, 53-62. English transl. in: Lithuanian Math. J. 23 (1983), 283-289. MR 85d:11083

[Lau84] A. Laurinchikas, The universality theorem. II (Russian), Litovsk. Mat. Sb. 24 (1984), no. 2, 113-121. English transl. in: Lithuanian Math. J. 24 (1984), 143-149. MR 86c: 11064

[Lau95] A. Laurinchikas, On the universality of the Riemann zeta-function (Russian), Liet. Mat. Rink. 35 (1995), 502-507. English transl. in: Lithuanian Math. J. 35 (1995), 399-402. MR 98c: 11086

[Lau96] A. Laurinčikas, Limit theorems for the Riemann zeta-function, Kluwer Acad. Publ., Dordrecht, 1996. MR 96m:11070

[Lau97] A. Laurinchikas, The universality of the Lerch zeta-function (Russian), Liet. Mat. Rink. 37 (1997), 367-375. English transl. in: Lithuanian Math. J. 37 (1997), 275280. MR 98k:11122

[Lau98a] A. Laurinchikas, On the Lerch zeta function with rational parameters (Russian), Liet. Mat. Rink. 38 (1998), 113-124. English transl. in: Lithuanian Math. J. 38 (1998) (to appear). CMP 99:06

[Lau98b] A. Laurinčikas, On the Matsumoto zeta-function, Acta Arith. 84 (1998), 1-16. CMP 98:10

$\left[\right.$ LaMa99 $\left.^{+}\right]$A. Laurinčikas and K. Matsumoto, The universality of zeta-functions attached to certain cusp forms, preprint.

[Leó99] F. León-Saavedra, Universal functions on the unit ball and the polydisk, Function spaces (Proc. Conf., Edwardsville, IL, 1998), Amer. Math. Soc., Providence, RI, 1999.

[LeMo97] F. León-Saavedra and A. Montes-Rodríguez, Linear structure of hypercyclic vectors, J. Funct. Anal. 148 (1997), 524-545. MR 98h:47028b

$2 \mathrm{~b}, 4 \mathrm{a}, 4 \mathrm{c}, 5$

$\left[\right.$ LeMo99 $\left.{ }^{+}\right]$F. León-Saavedra and A. Montes-Rodríguez, Spectral theory and hypercyclic subspaces, Trans. Amer. Math. Soc. (to appear). $\quad \mathbf{2 a , 2 b , 2 d , 4 a , 4 c , 5}$

[Lor53] G. G. Lorentz, Bernstein polynomials, Univ. of Toronto Press, Toronto, 1953. MR 15:217a

[Luh70] W. Luh, Approximation analytischer Funktionen durch überkonvergente Potenzreihen und deren Matrix-Transformierten, Mitt. Math. Sem. Giessen 88 (1970). MR 43:6411 
[Luh74a] W. Luh, Über die Anwendung von Übersummierbarkeit zur Approximation regulärer Funktionen, Topics in Analysis (Colloq. Math. Anal., Jyväskylä, 1970), 260-267, Springer, Berlin, 1974. MR 52:3492

$3 a, 4 d$

[Luh74b] W. Luh, Über die Summierbarkeit der geometrischen Reihe, Mitt. Math. Sem. Giessen 113 (1974). MR 56:910

[Luh76] W. Luh, Über den Satz von Mergelyan, J. Approx. Theory 16 (1976), 194-198. MR 55:671

4d

[Luh78] W. Luh, On universal functions, Fourier Analysis and Approximation Theory (Proc. Colloq., Budapest, 1976), Vol. II, 503-511, North-Holland, Amsterdam, 1978. MR 80m:30003

$2 \mathrm{a}, 3 \mathrm{a}, \mathbf{4 a}, \mathbf{4 b}$

[Luh79a] W. Luh, Über cluster sets analytischer Funktionen, Acta Math. Acad. Sci. Hungar. 33 (1979), 137-142. MR 80f:30022

$4 a, 4 b$

[Luh79b] W. Luh, Universalfunktionen in einfach zusammenhängenden Gebieten, Aequationes Math. 19 (1979), 183-193. MR 80m:30040 3a,4b

[Luh86] W. Luh, Universal approximation properties of overconvergent power series on open sets, Analysis 6 (1986), 191-207. MR 87m:30058

[Luh88] W. Luh, Holomorphic monsters, J. Approx. Theory 53 (1988), 128-144. MR 90a:30091

[Luh93] W. Luh, Universal functions and conformal mappings, Serdica 19 (1993), 161-166. MR 94k:30012

[Luh96] W. Luh, Entire functions with various universal properties, Complex Variables Theory Appl. 31 (1996), 87-96. MR 97m:30030

$4 \mathrm{a}, 4 \mathrm{c}$

[Luh97] W. Luh, Multiply universal holomorphic functions, J. Approx. Theory 89 (1997), 135-155. MR 98e:30043

$4 \mathrm{~b}, 4 \mathrm{c}, 4 \mathrm{~d}$

[LMM98] W. Luh, V. A. Martirosian, and J. Müller, T-universal functions with lacunary power series, Acta Sci. Math. (Szeged) 64 (1998), 67-79. MR 99f:30055 4a

[LMM99 ${ }^{+}$W. Luh, V. A. Martirosian, and J. Müller, Universal entire functions with gap power series, Indag. Math. (N.S.) (to appear).

$4 a, 4 b$

[LuLu85] S. Yu. Lukashenko and T. P. Lukashenko, The rate of growth of coefficients of universal series (Russian), Probability Theory, Theory of Random Processes, and Functional Analysis (Russian) (Proc. Conf., Moscow, 1984), 128-130, Moskov. Gos. Univ., Moscow, 1985. CMP 90:06

$3 \mathbf{c}$

[Mcc92] C. R. MacCluer, Chaos in linear distributed systems, J. Dynam. Systems Measurement Control 114 (1992), 322-324. Zbl 778:93038

[Mac52] G. R. MacLane, Sequences of derivatives and normal families, J. Analyse Math. 2 (1952/53), 72-87. MR 14:741d 2a,4c

[Mar35] J. Marcinkiewicz, Sur les nombres dérivés, Fund. Math. 24 (1935), 305-308. Zbl 11:107

$\left[\mathrm{MaPe} 99^{+}\right]$F. Martínez-Giménez and A. Peris, Hypercyclic and chaotic backward shift operators on Köthe echelon spaces, preprint.

2c, 2d,5

[Mat93] V. Matache, Notes on hypercyclic operators, Acta Sci. Math. (Szeged) 58 (1993), 401-410. MR 94k:47011

[Mat95] V. Matache, Spectral properties of operators having dense orbits, Topics in Operator Theory, Operator Algebras and Applications (Proc. Conf., Timişoara, 1994), 221237, Inst. Math. Roman. Acad., Bucharest, 1995. MR 98j:47002

[Mth94] V. Mathew, A note on hypercyclic operators on the space of entire sequences, Indian J. Pure Appl. Math. 25 (1994), 1181-1184. MR 95i:47055 4c,5

[Maz37] S. Mazurkiewicz, Sur l'approximation des fonctions continues d'une variable réelle par les sommes partielles d'une série de puissances, C. R. Soc. Sci. Lett. Varsovie Cl. III 30 (1937), 25-30. Zbl 17:204

$\mathbf{1 c}, \mathbf{3 a}, \mathbf{4 d}$

$\left[\mathrm{MeNe} 99^{+}\right]$A. Melas and V. Nestoridis, Universality of Taylor series as a generic property of holomorphic functions, preprint.

$3 \mathrm{c}, 4 \mathrm{~d}$

[MNP97] A. Melas, V. Nestoridis, and I. Papadoperakis, Growth of coefficients of universal Taylor series and comparison of two classes of functions, J. Anal. Math. 73 (1997), 187-202. CMP 98:10

$4 d$

[Men45] D. Menchoff, Sur les séries trigonométriques universelles, C. R. (Dokl.) Acad. Sci. URSS (N.S.) 49 (1945), 79-82. MR 7:435e 
[Men47] D. E. Men'shov, On the partial sums of trigonometric series (Russian), Mat. Sb. (N.S.) 20(62) (1947), 197-238. MR 8:577a 1c,3c

[Men48] D. Men'shov, On the convergence in measure of trigonometric series (Russian), Dokl. Akad. Nauk SSSR (N.S.) 59 (1948), 849-852. MR 9:426b 3c

[Men50] D. E. Men'shov, On convergence in measure of trigonometric series (Russian), Trudy Mat. Inst. Steklov. 32 (1950). English transl. in: Amer. Math. Soc. Transl. (1) 3 (1962), 196-270. MR 12:254g

[Men54] D. E. Men'shov, On the limits of indeterminateness of partial sums of universal trigonometric series (Russian), Moskov. Gos. Univ. Uchen. Zap. 165 Mat. 7 (1954), 3-33. English transl. in: Amer. Math. Soc. Transl. (2) 111 (1978), 1-33. MR 16:467a

[Men63] D. Men'shov, On universal sequences of functions (Russian), Dokl. Akad. Nauk SSSR 151 (1963), 1283-1285. English transl. in: Soviet Math. Dokl. 4 (1963), 1191-1193. MR 27:3967

3c

[Men64] D. E. Men'shov, Universal sequences of functions (Russian), Mat. Sb. (N.S.) 65(107) (1964), 272-312. English transl. in: Amer. Math. Soc. Transl. (2) 111 (1978), 81-116. MR 31:305

$3 \mathbf{c}$

[Mil93] J. B. Miller, Generalized functions for the Laplace transform and universal approximants, Generalized Functions and Their Applications (Proc. Symp., Varanasi, 1991), 131-140, Plenum, New York, 1993. MR 94h:46065

$4 a$

[MiMi99] T. L. Miller and V. G. Miller, Local spectral theory and orbits of operators, Proc. Amer. Math. Soc. 127 (1999), 1029-1037. MR 99f:47007

[Mil97] V. G. Miller, Remarks on finitely hypercyclic and finitely supercyclic operators, Integral Equations Operator Theory 29 (1997), 110-115. MR 98i:47017 2c,2d

[Mon96a] A. Montes-Rodríguez, A note on Birkhoff open sets, Complex Variables Theory Appl. 30 (1996), 193-198. MR 97h:30056

[Mon96b] A. Montes-Rodríguez, Banach spaces of hypercyclic vectors, Michigan Math. J. 43 (1996), 419-436. MR 98g:47027 1c,2b,4a,5

[Mon97] A. Montes-Rodríguez, Vector spaces of universal functions, Complex Methods in Approximation Theory (Proc. Workshop, Almería, 1995), 113-116, Univ. Almería, Almería, 1997. MR 99c:30067 2b,4a,5

[Mon98a] A. Montes-Rodríguez, Composition operators and hypercyclic vectors, Studies on Composition Operators (Proc. Conf., Laramie, WY, 1996), 157-165, Amer. Math. Soc., Providence, RI, 1998. MR 98k:47063

$2 \mathrm{~b}, \mathbf{4 a}$

[Mon98b] A. Montes-Rodríguez, A Birkhoff theorem for Riemann surfaces, Rocky Mountain J. Math. 28 (1998), 663-693. CMP 99:03

[Nes96] V. Nestoridis, Universal Taylor series, Ann. Inst. Fourier (Grenoble) 46 (1996), 1293-1306. MR 97k:30001

$1 \mathrm{c}, 3 \mathrm{c}, 4 \mathrm{~d}$

$\left[\mathrm{Nes}^{+}{ }^{+}\right]$V. Nestoridis, An extension of the notion of universal Taylor series, Computational Methods and Function Theory 1997 (Proc. Conf., Nicosia, 1997) (to appear). 3c,4d

[Pál14] J. Pál, Zwei kleine Bemerkungen, Tôhoku Math. J. 6 (1914/15), 42-43. JFM 45:634

$1 \mathrm{~d}, 3 \mathrm{a}$

[Pál15] G. Pál, On some generalisations of a theorem of Weierstrass (Hungarian), Math. Phys. Lapok 24 (1915), 243-247. JFM 45:634 3a

[Pav92] M. Pavone, Chaotic composition operators on trees, Houston J. Math. 18 (1992), 47-56. MR 93c: 47038

[Per98] A. Peris, Personal communications, $1998 . \quad \mathbf{1 b}, \mathbf{2 b}$

$\left[\right.$ Per99 $\left.{ }^{+}\right]$A. Peris, Chaotic polynomials on Fréchet spaces, Proc. Amer. Math. Soc. (to appear). CMP 98:09

$2 \mathrm{~d}, 4 \mathrm{c}$

[Pog75] N. B. Pogosyan, Representation of measurable functions by orthogonal series (Russian), Mat. Sb. (N.S.) 98(140) (1975), no. 1, 102-112. English transl. in: Math. USSR-Sb. 27 (1975), 93-102. MR 58:6901a

[Pog76] N. B. Pogosyan, Representation of measurable functions by bases in $L_{p}[0,1],(p \geq 2)$ (Russian), Akad. Nauk Armyan. SSR Dokl. 63 (1976), 205-209. MR 58:6901b 3c

[PrAz92] V. Protopopescu and Y. Y. Azmy, Topological chaos for a class of linear models, Math. Models Methods Appl. Sci. 2 (1992), 79-90. MR 93c:58126 3e,5

[Rea88] C. J. Read, The invariant subspace problem for a class of Banach spaces. II. Hypercyclic operators, Israel J. Math. 63 (1988), 1-40. MR 90b:47013 2b 
[Rei77] A. Reich, Universelle Werteverteilung von Eulerprodukten, Nachr. Akad. Wiss. Göttingen Math.-Phys. Kl. II 1977, 1-17. MR 58:27862 6

[Rei80] A. Reich, Werteverteilung von Zetafunktionen, Arch. Math. 34 (1980), 440-451. MR 82b:10051

[Rei82] A. Reich, Zur Universalität und Hypertranszendenz der Dedekindschen Zetafunktion, Abh. Braunschweig. Wiss. Ges. 33 (1982), 197-203. MR 84h:12016

[Rol69] S. Rolewicz, On orbits of elements, Studia Math. 32 (1969), 17-22. MR 39:3292

[Sal91] H. Salas, A hypercyclic operator whose adjoint is also hypercyclic, Proc. Amer. Math. Soc. 112 (1991), 765-770. MR 91j:47016 2a,2c,2d,5

[Sal95] H. N. Salas, Hypercyclic weighted shifts, Trans. Amer. Math. Soc. 347 (1995), 9931004. MR 95e: 47042

$\left[\mathrm{Sal}^{+}\right] \quad$ H. Salas, Supercyclicity and weighted shifts, preprint.

[Scm97] C. Schmoeger, On the norm-closure of the class of hypercyclic operators, Ann. Polon. Math. 65 (1997), 157-161. MR 98b:47050

[Scn97] I. Schneider, Schlichte Funktionen mit universellen Approximationseigenschaften, Mitt. Math. Sem. Giessen 230 (1997). MR 98c:30022

[SeWa41] W. Seidel and J. L. Walsh, On approximation by euclidean and non-euclidean translations of an analytic function, Bull. Amer. Math. Soc. 47 (1941), 916-920. MR 4:10a

[Sel51] A. I. Seleznev, On universal power series (Russian), Mat. Sb. (N.S.) 28(70) (1951), 453-460. MR 13:23e 3a,4d

[SeDo77] A. I. Seleznev and L. K. Dodunova, Some classes of universal series (Russian), Izv. Vyssh. Uchebn. Zaved. Mat. 1977, no. 12(187), 92-98. English transl. in: Soviet Math. (Iz. VUZ) 21 (1977), no. 12, 69-73. MR 58:17056

[SeDo82] A. I. Seleznev and L. K. Dodunova, On two theorems of A. F. Leontev on the completeness of subsystems of Faber and Jacobi polynomials (Russian), Izv. Vyssh. Uchebn. Zaved. Mat. 1982, no. 4, 51-55. English transl. in: Soviet Math. (Iz. VUZ) 26 (1982), no. 4, 60-66. MR 84j:30061

[SMV77] A. I. Seleznev, I. V. Motova, and V. A. Volokhin, The completeness of systems of functions and universal series (Russian), Izv. Vyssh. Uchebn. Zaved. Mat. 1977, no. 11(186), 84-90. English transl. in: Soviet Math. (Iz. VUZ) 21 (1977), no. 11, 70-75. MR 58:22496

$1 \mathrm{~d}, 4 \mathrm{~d}$

[Sha93] J. H. Shapiro, Composition operators and classical function theory, Springer, New York, 1993. MR 94k:47049 1b,2c,2d,4a

[Shk93] S. A. Shkarin, On the growth of D-universal functions (Russian), Vestnik Moskov. Univ. Ser. I Mat. Mekh. 1993, no. 6, 80-83. English transl. in: Moscow Univ. Math. Bull. 48 (1993), no. 6, 49-51. MR 96i:30022

[Sie38] W. Sierpiński, Sur une série de puissances universelle pour les fonctions continues, Studia Math. 7 (1938), 45-48. Zbl 18:114 1d,3a

[Söv77] A. Sövegjártó, A note on universal elements (Hungarian), Mat. Lapok 28 (1977/80), 327-328. MR 82f: 47044

[Str81] K. R. Stromberg, Introduction to classical real analysis, Wadsworth International, Belmont, CA, 1981. MR 82c:26002

[Tal57] A. A Talalyan, On the convergence almost everywhere of subsequences of partial sums of general orthogonal series (Russian), Akad. Nauk Armyan. SSR Izv. Fiz.Mat. Estest. Tekhn. Nauki 10 (1957), no. 3, 17-34. MR 19:742b 1d,3c,3d

[Tal59a] A. A. Talalyan, Universal orthogonal series (Russian), Izv. Akad. Nauk Armyan. SSR Ser. Fiz.-Mat. Nauk 12 (1959), no. 1, 27-42. MR 21:5110 3c

[Tal59b] A. A. Talalyan, On series in bases of $L_{p}$ space which are universal with respect to permutations (Russian), Akad. Nauk Armyan. SSR Dokl. 28 (1959), 145-150. MR 22:9853

[Tal60a] A. A. Talalyan, On the convergence and summability almost everywhere of general orthogonal series (Russian), Izv. Akad. Nauk Armyan. SSR Ser. Fiz.-Mat. Nauk 13 (1960), no. 2, 31-61. MR 27:2787

3d

[Tal60b] A. A. Talalyan, Series universal with respect to rearrangement (Russian), Izv. Akad. Nauk SSSR Ser. Mat. 24 (1960), 567-604. MR 22:9854 
[Tal60c] A. A. Talalyan, The representation of measurable functions by series (Russian), Uspekhi Mat. Nauk 15 (1960), no. 5(95), 77-141. English transl. in: Russian Math. Surveys 15 (1960), no. 5, 75-136. MR 23:A2704 3c,3d

[ThTo85] B. Thorpe and L. Tomm, Universal approximation by regular weighted means, Pacific J. Math. 117 (1985), 443-455. MR 86f:30002

[TiLi95] L. X. Tian and Z. R. Liu, Nonwandering operators in infinite-dimensional linear spaces (Chinese), Acta Math. Sci. (Chinese) 15 (1995), 455-460. MR 97e:47012 2d

[TiLu96] L. Tian and D. Lu, The property of nonwandering operator (Chinese), Appl. Math. Mech. 17 (1996), 151-156. English transl. in: Appl. Math. Mech. (English Ed.) 17 (1996), 155-161. MR 97a:47029

$2 d$

[Tod85] V. T. Todorov, Linear differential operators are transitive, Godishnik Vissh. Uchebn. Zaved. Prilozhna Mat. 21 (1985), no. 4, 17-24. MR 88i:58014

[ToKo80] V. Todorov and O. Korov, An infinitely differentiable function, the set of derivatives of which is everywhere dense (Russian), Mathematics and Education in Mathematics (Bulgarian) (Proc. Conf., Sunny Beach, 1980), 91-94, B"'lg. Akad. Nauk., Sofia, 1980. Zbl 573:26014

[ToTr82] L. Tomm and R. Trautner, A universal power series for approximation of measurable functions, Analysis 2 (1982), 1-6. MR 85e:30004

$\mathbf{4 d}$

[Tuy59] Kh. Tŭ [H. Tuy], The structure of measurable functions (Russian), Dokl. Akad. Nauk SSSR 126 (1959), 37-40. MR 21:5000

[Tuy60] Kh. Tuı̆ [H. Tuy], The "universal primitive" of J. Marcinkiewicz (Russian), Izv. Akad. Nauk SSSR Ser. Mat. 24 (1960), 617-628. MR 22:3770

[Vor75a] S. M. Voronin, A theorem on the distribution of values of the Riemann zeta-function (Russian), Dokl. Akad. Nauk SSSR 221 (1975), 771. English transl. in: Soviet Math. Dokl. 16 (1975), 410. MR 52:3079

[Vor75b] S. M. Voronin, A theorem on the "universality" of the Riemann zeta-function (Russian), Izv. Akad. Nauk SSSR Ser. Mat. 39 (1975), 475-486. English transl. in: Math. USSR-Izv. 9 (1975), 443-453. MR 57:12419

[Vor77] S. M. Voronin, Analytic properties of the Dirichlet generating series of arithmetic objects (Russian), Thesis, Moscow, 1977.

[Vor84] S. M. Voronin, On the distribution of zeros of some Dirichlet series (Russian), Trudy Mat. Inst. Steklov. 163 (1984), 74-77. English transl. in: Proc. Steklov Inst. Math. 163 (1985), 89-92. MR 86g:11048

[Web95] G. F. Webb, Periodic and chaotic behavior in structured models of cell population dynamics, Recent Developments in Evolution Equations (Proc. Int. Seminar, Glasgow, 1994), 40-49, Longman Scientific and Technical, Harlow, 1995. MR 98a:92006

$\mathbf{2 a}, \mathbf{3 e}$

[Zap88] P. Zappa, On universal holomorphic functions, Boll. Un. Mat. Ital. A (7) 2 (1988), 345-352. MR 90g:30039

Fachbereich Mathematik, Fernuniversität Hagen, 58084 Hagen, Germany

E-mail address: kg.grosse-erdmann@fernuni-hagen.de 